\title{
以水为溶剂的铃木-宫浦偶联反应最新研究进展
}

\author{
徐 鹏段新红* \\ (北京林业大学理学院 北京 100083)
}

\begin{abstract}
摘要 过渡金属催化的铃木-宫浦偶联反应是目前构建碳-碳键最为重要的有机手段之一, 人们对以水为溶剂的绿色有 机反应日益重视, 在水介质中进行铃木-宫浦偶联反应已成为人们研究和开发的热点. 就近年来在水介质中进行均相 和非均相催化铃木-宫浦偶联反应的进展加以综述, 并展望了其应用前景.
\end{abstract}

关键词＼cjkstart铃木-宫浦偶联; 水为溶剂; 非均相催化; 均相催化

\section{Recent Progress in the Suzuki-Miyaura Cross-Coupling Reactions in Water}

\author{
$\mathrm{Xu}$, Peng Duan, Xinhong* \\ (College of Science, Beijing Forestry University, Beijing 100083)
}

\begin{abstract}
Transition-metal-catalyzed Suzuki-Miyaura cross-coupling is one of the most powerful transformations for C-C biaryl bond formation at present. With the increasing demand for green chemistry, water as solvent for the Suzuki-Miyaura cross-coupling reactions has been of widespread interest. The literature in recent years on the Suzuki-Miyaura cross-coupling reactions by heterogeneous or homogeneous catalysis in water is reviewed, and their perspectives for further developments are also presented.

Keywords Suzuki-Miyaura cross-coupling; in water; heterogeneous catalysis; homogeneous catalysis
\end{abstract}

过渡金属催化的碳-碳偶联反应一直以来是现代有 机领域最为重要的合成工具之一, 正因为联芳烃是药 物、天然产物和催化剂配体等复杂化合物中的核心结构, 使得芳基的碳一碳偶联反应成为此领域的研究和开发热 点, 同时亦是一个极具挑战性的课题 ${ }^{[1]} .1981$ 年, 铃木 章等 ${ }^{[2]}$ 采用有机硼试剂与芳基卤代烃进行偶联反应, 成 功制备了联苯化合物, 即铃木-宫浦偶联反应(SuzukiMiyaura cross-coupling, SMC). 随后, 在 SMC 反应的不 断开发和利用过程中人们发现，该反应的条件较温和， 具有较强的底物适用性以及官能团容忍度, 解决了芳香 族之间的碳一碳交叉偶联困难及副产物多等突出难题 ${ }^{[3]}$, 例如: 该反应不仅能用于合成复杂的联苯衍生物及液晶 材料等, 而且是合成众多天然产物及药物的关键步骤 ${ }^{[4]}$. 另一方面, 以水为溶剂进行化学反应不仅具有环境友 好、无毒及价格便宜等优势, 而且能提高化学反应的反 应速度和选择性, 因此, 以水为溶剂的绿色化学反应也
是化学家们关注的焦点 ${ }^{[5]}$. 由于 SMC 反应的底物卤代 芳烃和嗍化试剂(尤其是芳基硼酸)在水中具有良好的稳 定性这一优势, 为水介质中进行该反应开辟了可能性. 近二十年来, 以水为溶剂的 SMC 反应研究得到了突飞 猛进的发展，尤其是在 2010 2016 年期间, Polshet$\operatorname{tiwar}^{[6]} 、 \mathrm{Navarro}^{[7 \mathrm{a}]}$ 和 Ward 等 ${ }^{[7 b]}$ 分别对这一领域的进展 进行了详细的综述.

就近几年来以水为溶剂的 SMC 反应进展进行了综 述, 主要分为非均相催化和均相催化反应两个部分, 并 探讨了不同的催化剂结构对 SMC 反应产生的影响，包 括以下几点: (1)反应活性及产率; (2)反应条件; (3)底物 的普适性; (4)官能团耐受性; (5)催化剂的重复使用能力.

\section{1 非均相催化 SMC 反应}

非均相催化反应因催化剂用量少、易回收并能再利 用以及产品易分离等优势备受科学家青睐 ${ }^{[8]}$, 同时也是

\footnotetext{
* Corresponding author. E-mail: xinhong116@163.com Received August 12, 2019; revised October 10, 2019; published online November 13, 2019 Project supported by the National Natural Science Foundation of China (No. 21671021). 国家自然科学基金(No. 21671021)资助项目.
} 
实现绿色化学反应的重要途径, 尤其是在水中进行非均 相催化反应更是有机界广泛关注的焦点 ${ }^{[9]}$.

\section{1 负载型 $\mathrm{Pd}$ 催化剂}

近年来, 使用不同类型的载体固定钯催化剂并在水 介质中进行非均相 SMC 反应的研究已掀起了热潮. 由 于载体的表面官能团和电子特性等均对钯催化剂的稳 定性、催化活性、选择性以及寿命影响很大, 因此, 人 们在不断探索新型催化剂的同时, 也在致力于发掘优质 而高效的载体.

采用化学键将催化剂固定在载体上, 可使每一金属 活性中心均处于反应分子可以触及的载体表面, 提高催 化剂活性; 而且 “化学键联”不仅避免活性组分流失, 更 利于催化剂的回收.

2016 年, Gogoi 课题组 ${ }^{[10]}$ 以环保易得的纳米硅胶为 载体，通过 “Linker” 将 2-羟基苯席夫碱的钯(II)配合物 固定在硅胶上, 制得了一种新型四配位聚合物 Pd Schiff base complex (Scheme 1). 在 $50{ }^{\circ} \mathrm{C}$ 下, $6.8 \mathrm{~mol} \%$ 该催化 剂以高收率 $(94 \% \sim 100 \%)$ 顺利催化溴代芳烃; 虽然低活 性氯代芳烃也能实现 SMC 反应, 但一则需要在混合溶 剂中进行 $\left(\mathrm{DMF} / \mathrm{H}_{2} \mathrm{O}, V: V=1: 1\right)$, 二则产率明显降低 $(62 \% \sim 85 \%)$. 此外, 该催化剂可重复使用 6 次, 但产率 从 98\%降到 90\%, 催化活性略有降低.
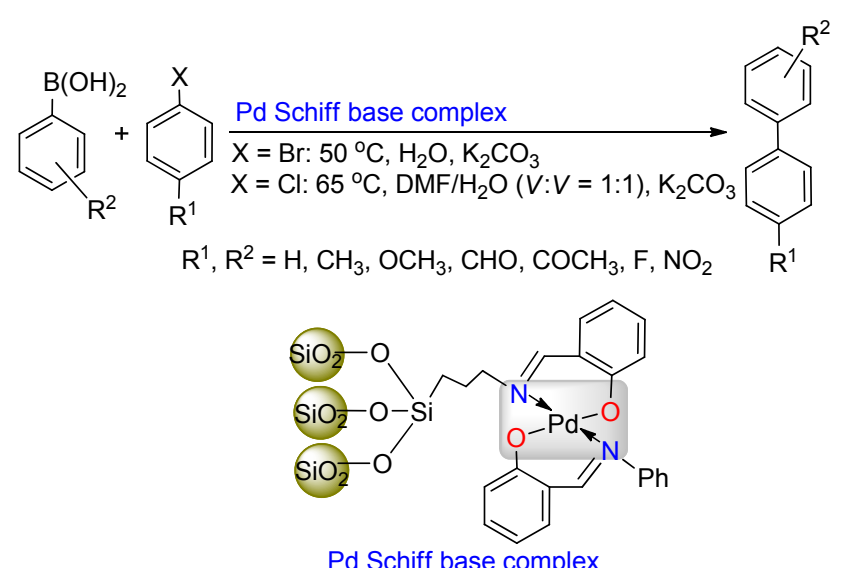

图式 $1 \mathrm{Pd}$ 席夫碱配合物催化 SMC 反应 Scheme 1 Pd Schiff base complex catalyzed SMC reactions

2017 年, Rhee 等 ${ }^{[11]}$ 采用同样的方法, 将 2-吡啶席夫 碱钯(II)配合物固定在硅胶上，再经 $\mathrm{NaBH}_{4}$ 还原得到一 种新型二齿配位聚合物 APPd(0)@Si (Scheme 2). 与 Pd 席夫碱配合物相比，该催化剂的用量少 $(1 \mathrm{~mol} \%)$, 却能 高效催化澳代芳烃的反应(最高产率可达 99\%)，且官能 团耐受性更好. 尽管对氯代芳烃没有催化活性，但其重 复使用能力更为优异，连续使用 8 次后催化活性从 $99 \%$ 仅降到 $95 \%$.

介孔碳是近年来迅速发展起来的一种多孔型材料,
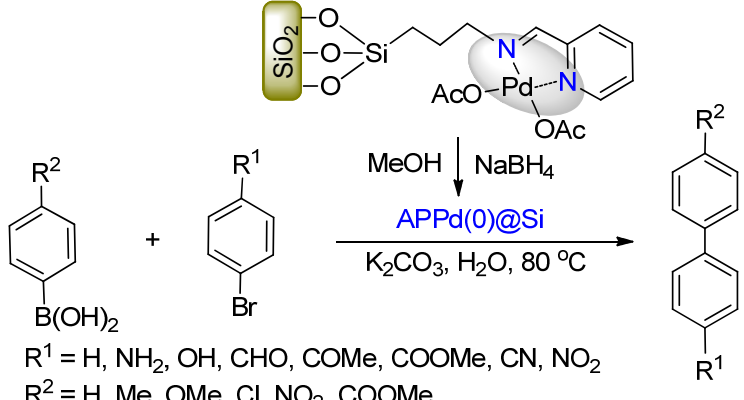

$\mathrm{R}^{2}=\mathrm{H}, \mathrm{Me}, \mathrm{OMe}, \mathrm{Cl}, \mathrm{NO}_{2}, \mathrm{COOMe}$

图式 $2 \mathrm{APPd}(0) @ \mathrm{Si}$ 催化 SMC 反应

Scheme 2 APPd(0)@Si catalyzed SMC reactions

由于具有比表面和孔容大以及化学反应惰性等性能，目 前已作为优质载体被广泛用于非均相催化领域.

2018 年, 朱大建课题组 ${ }^{[12]}$ 就以 5,10,15,20-苯基卟啉 (TPP)的聚合物为载体, 经 “一锅法” 实现 TPP 烷基化 的同时进行配合反应，将 $\mathrm{PdCl}_{2}$ “嵌” 入 $\mathrm{TPP}$, 成功制备 了一种新型的介孔型四配位纳米聚合物 Pd/KAPs(DCMTPP) (Scheme 3). 这一独特结构的催化剂(1.29 mol\%)在 $100{ }^{\circ} \mathrm{C}$ 下反应表现出高效的 $\mathrm{SMC}$ 反应活性: 碘或澳代 芳烃的产率都大于 $96 \%$, 而且 4-甲基氯苯的产率也能达 到 93\%. 其另一个优点是催化剂重复使用 5 次后产率仅 从 99\%降到 96\%, 基本保持了催化活性.

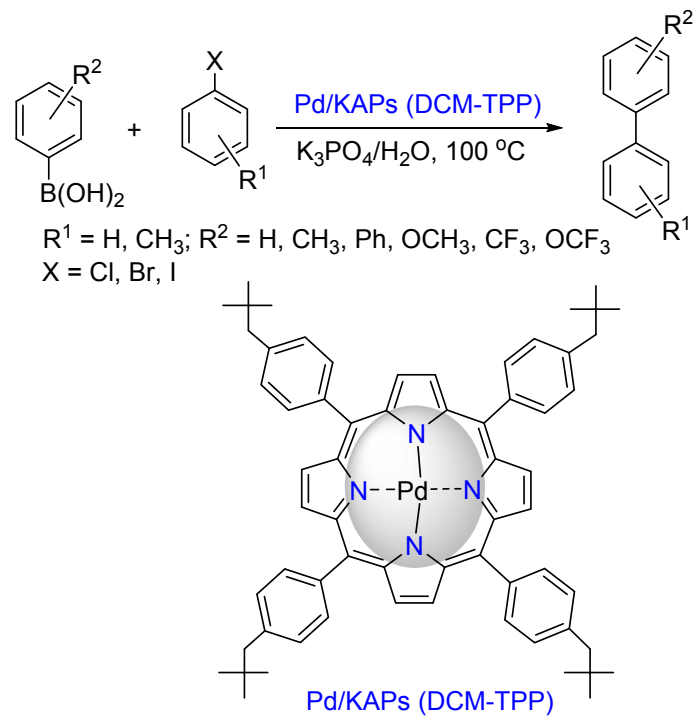

图式 $3 \mathrm{Pd} / \mathrm{KAPs}(\mathrm{DCM}-\mathrm{TPP})$ 催化 SMC 反应

Scheme $3 \mathrm{Pd} / \mathrm{KAPs}(\mathrm{DCM}-\mathrm{TPP})$ catalyzed SMC reactions

Jadhav 等 ${ }^{[13]}$ 以廉价的 $D$-葡萄糖为前体, 经系列处 理后形成含有 $\mathrm{COOH}$ 及 $\mathrm{SO}_{3} \mathrm{H}$ 活性基团的多环芳烃碳质 材料, 并以此为载体与 $\mathrm{PdCl}_{2}$ 进一步络合制得一种新型 的介孔型二齿配位聚合物 $\mathrm{Pd} @ \mathrm{CC}-\mathrm{SO}_{3} \mathrm{H}-\mathrm{NH}_{2}$ (Scheme 4). 与 $\mathrm{Pd} / \mathrm{KAPs}(\mathrm{DCM}-\mathrm{TPP})$ 的催化活性接近, 质量分数 为 $10 \%$ 的该催化剂能顺利活化碘、溴及氯代芳烃，最高 产率可达 96\%; 同样，该催化剂表现出类似的重复使用 
率：连续使用 5 次后产率从 96\%仅降至 94\%, 基本维持 原有的催化活性。

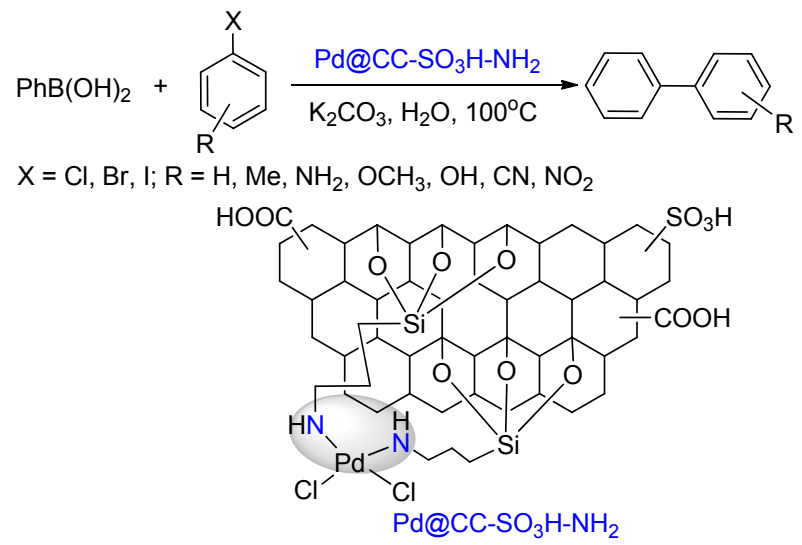

图式 $4 \mathrm{Pd} @ \mathrm{CC}-\mathrm{SO}_{3} \mathrm{H}-\mathrm{NH}_{2}$ 催化 $\mathrm{SMC}$ 反应

Scheme $4 \mathrm{Pd} @ \mathrm{CC}-\mathrm{SO}_{3} \mathrm{H}-\mathrm{NH}_{2}$ catalyzed SMC reactions

介孔型分子篮 SBA-15 同样是科学家关注的一类新 型载体. 2016 年, Rostamnia 课题组 ${ }^{[14]}$ 就将三乙烯二胺 (DABCO)与 3-氯丙基三甲氧基硅烷反应所产生的双正 电荷产物以共价键形式固定在 SBA-15 上, 并利用这一 双正电荷的特性进一步与 $\mathrm{PdCl}_{2}$ 络合, 成功制备了一种

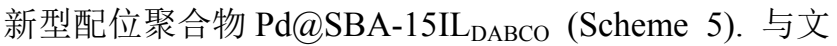
献 $[12,13]$ 相比，该催化剂用量更少，仅为 $0.5 \mathrm{~mol} \%$; 对 碘及溴代芳烃的催化活性更高, SMC 反应产率均达 97\% 98\%．尽管该催化剂对氯代芳烃没有催化活性, 但重复使用能力更加优秀，连续使用 9 次后产率从 $97 \%$ 仅降至 93\%，基本保持原有催化活性.

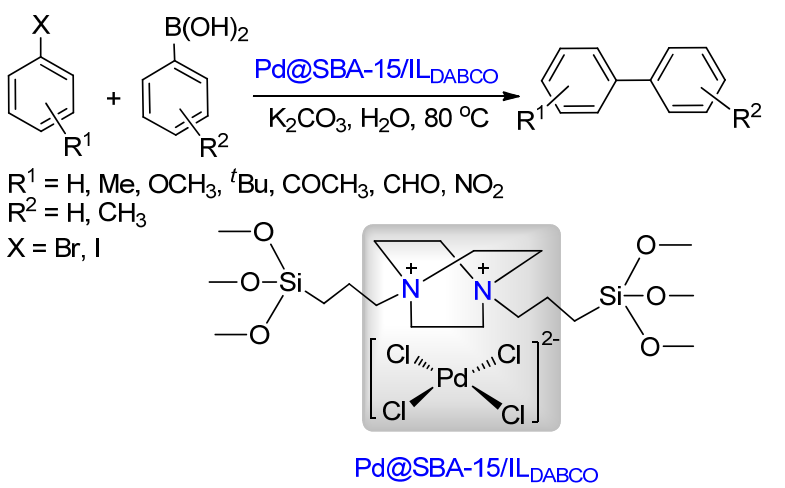

图式 $5 \mathrm{Pd} @ \mathrm{SBA}-15 / \mathrm{IL}_{\mathrm{DABCO}}$ 催化 $\mathrm{SMC}$ 反应 Scheme 5 Pd@SBA-15/ $\mathrm{IL}_{\mathrm{DABCO}}$ catalyzed SMC reactions

另一方面, 利用多孔性载体的强吸附力固定催化剂 也是制备负载型催化剂的方法之一, 由于这一类型载体 负载量大, 催化剂分散度高, 因而能较大幅度地提高催 化剂的催化性能. 相较于 “化学键联” 法, 吸附法的牢 固性较差, 因此催化剂的重复使用率相对较低.

2019 年, López 课题组 ${ }^{[15]}$ 利用介孔型硅胶为载体吸 附并包裹纳米钯 $(\mathrm{Pd} N \mathrm{NP})$, 制备了一种新型中空微球催
化剂 Pd-Cap (Scheme 6). 研究表明，该催化剂具有毒性 小、水中稳定性好的突出优势, 尤其是在生理温度下 $\left(37{ }^{\circ} \mathrm{C}\right)$ 能顺利催化 4-碘苯甲醛与 2-噻吩硼酸的 SMC 反 应，产率达 $86 \%$. 区别于常规 SMC 反应所必需的碱性 条件，该反应是在 PBS 溶液( $\mathrm{pH}$ 7.2)中进行的，这些独 特的反应优势为生理环境下实施 SMC 反应制备药物提 供了可能. 但相比之下，该催化剂用量大(10 $\mathrm{mol} \%)$, 活 性低、对溴和氯代芳烃没有催化活性，底物的适用性及 官能团耐受性需要进一步研究. 该催化剂能否重复使用 并未加报道.

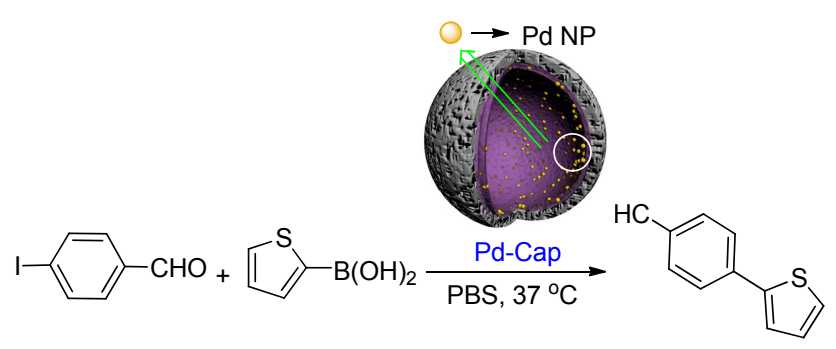

图式 6 Pd-Cap 催化 SMC 反应

Scheme 6 Pd-Cap catalyzed SMC reactions

Lee 等 ${ }^{[16]}$ 抛开常规载体, 以非定形结构的真黑素 (eumelanin)为载体, 利用其对钯(II)的强大吸附力制备 了一种新型纳米催化剂 Eum@PdNPs (Scheme 7). 相较 于 Pd-Cap, 该催化剂在 $80{ }^{\circ} \mathrm{C}$ 下表现出强大的催化活性, 仅 $0.05 \mathrm{~mol} \%$ 用量就能顺利催化溴和氯代芳烃, SMC 反 应产率最高可达 $99 \%$. 研究同时表明, 当氯代芳烃连有 推电子基及邻位基团时都不利于 SMC 反应进行. 该催 化剂的重复使用能力并无报道.

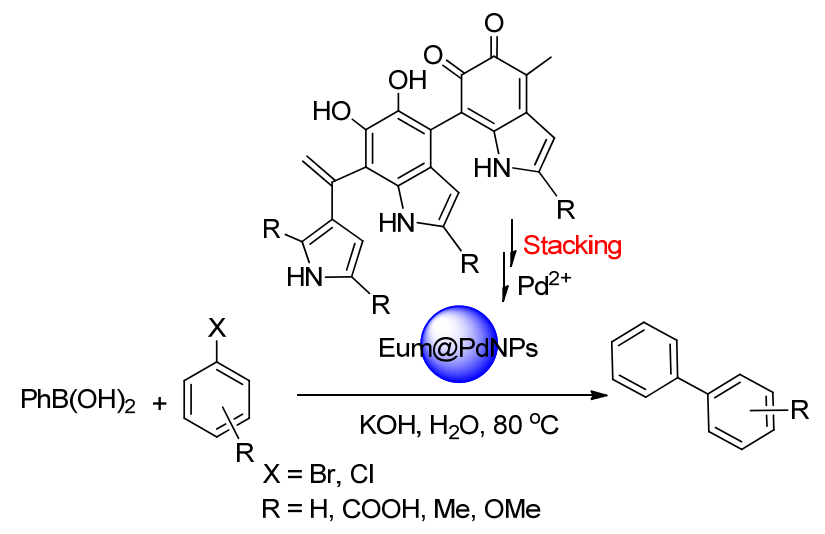

图式 7 Eum@Pd NPs 催化 SMC 反应

Scheme 7 Eum@Pd NPs catalyzed SMC reactions

\section{$1.2 \mathrm{Pd}$-配合物催化剂}

目前, 在水中进行非均相催化反应最为简单和直接 的手段就是使用不溶于水的金属配合物作为催化剂.

2018 年, Mösch-Zanetti 课题组 ${ }^{[17]}$ 就开发了新型双核 钯配合物 $\{\mathrm{PdCl}(\mathrm{S}-\mathrm{Phoz})\}_{2}$ 和 $\{\mathrm{PdI}(\mathrm{S}-\mathrm{Phoz})\}_{2}$ (Scheme 8). 
这一系列催化剂对 SMC 反应表现出优异的催化活性: 在 $90{ }^{\circ} \mathrm{C}$ 下, 仅 $0.01 \mathrm{~mol} \%$ 的 $\{\mathrm{PdCl}(\mathrm{S}-\mathrm{PhOZ})\}_{2}$ 或 $\{\mathrm{PdI}(\mathrm{S}-$ $\mathrm{PhOZ})\}_{2}$ 就能顺利催化溴代芳烃, SMC 产率均大于 $99 \%$. 但底物的适用性和官能团耐受性都比较差, 而且对氯代 芳烃也不具备催化活性. 这一类型催化剂能否循环使用 也没有报道。

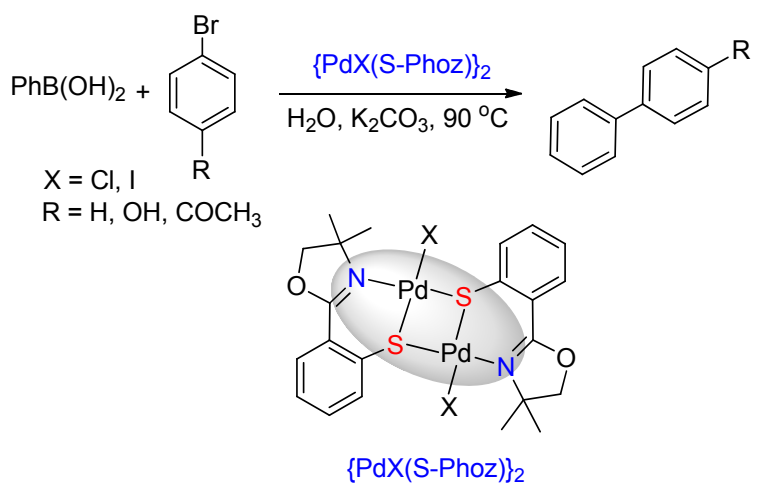

图式 $8\{\mathrm{PdX}(\mathrm{S}-\mathrm{PhOZ})\}_{2}$ 催化 $\mathrm{SMC}$ 反应

Scheme $8\{\mathrm{PdX}(\mathrm{S}-\mathrm{PhOZ})\}_{2}$ catalyzed SMC reactions

Asadi 课题组 ${ }^{[18]}$ 则探索了一种以双亚胺为配体的纳 米级钯配合物 (Scheme 9). 在 $80{ }^{\circ} \mathrm{C}$ 下该催化剂 $(0.2$ $\mathrm{mol} \%$ )不仅高效催化碘及澳代芳烃与苯硼酸的 SMC 反 应(产率 82\% 96\%); 而且还能顺利活化氯代芳烃(产率 均大于 $70 \%$ ), 官能团耐受性较好. 此外, 催化剂可循环 使用 6 次，产率从 95\%降至 90\%，催化活性略有降低.

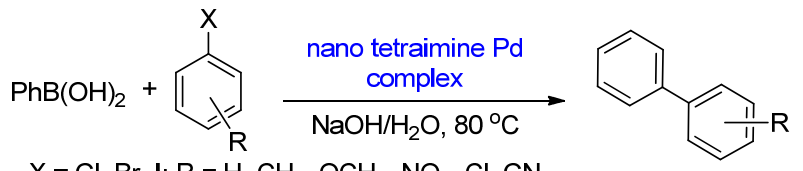

$\mathrm{X}=\mathrm{Cl}, \mathrm{Br}, \mathrm{I} ; \mathrm{R}=\mathrm{H}, \mathrm{CH}_{3}, \mathrm{OCH}_{3}, \mathrm{NO}_{2}, \mathrm{Cl}, \mathrm{CN}$

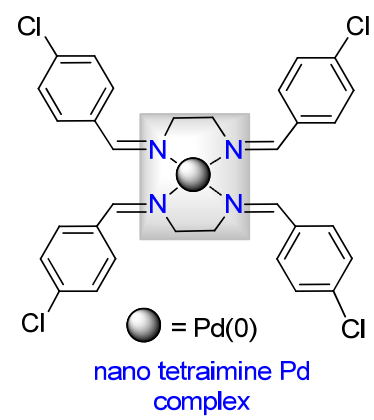

图式 9 纳米级双亚胺钯配合物催化 SMC 反应

Scheme 9 Nano tetraimine Pd complex catalyzed SMC reactions

\section{$1.3 \mathrm{Pd}-\mathrm{Fe}$ 磁性纳米催化剂}

近年来，利用磁性纳米催化剂进行非均相反应是科 学家研究的新热点. 在对 SMC 反应不断研究进程中, 涌现出许多将优异磁性功能纳米粒子(例如 $\mathrm{Fe}_{4} \mathrm{O}_{3}$ ) 与 $\mathrm{SMC}$ 催化剂相结合的新型催化剂.
2018 年, Chatterjee 等 ${ }^{[19]}$ 将纳米级 $\mathrm{Fe}_{3} \mathrm{O}_{4}$ 与 $\mathrm{PdCl}_{2}$ 在 高温下直接混合, 制备了一种新型磁性纳米催化剂 Pd$\gamma$ - $\mathrm{Fe}_{2} \mathrm{O}_{3}$ (Scheme 10). 在 $80{ }^{\circ} \mathrm{C}$ 及无配体参与下，该催化 剂顺利催化碘和溴代芳烃的 SMC 反应，产率可达 $89 \%$ ～98\%，且官能团耐受性好，但对氯代芳烃没有催 化活性. 值得注意的是，催化剂可通过磁性分离就能简 单回收，但在重复使用 6 次后产率从 $97 \%$ 降至 $89 \%$, 催 化活性有较为明显的降低.

$$
\begin{aligned}
& \mathrm{Fe}(\text { acac })_{3}+\left(\mathrm{NH}_{4}\right)_{2} \mathrm{Fe}\left(\mathrm{SO}_{4}\right)_{2} \cdot 6 \mathrm{H}_{2} \mathrm{O} \frac{\mathrm{NaOH}, \mathrm{H}_{2} \mathrm{O}}{180{ }^{\circ} \mathrm{C}, 48 \mathrm{~h}} \text { nano- } \mathrm{Fe}_{3} \mathrm{O}_{4} \\
& \frac{\mathrm{PdCl}{ }_{2}, \mathrm{H}_{2} \mathrm{O}}{180^{\circ} \mathrm{C}, 48 \mathrm{~h}}
\end{aligned}
$$

图式 $10 \mathrm{Pd}-\gamma-\mathrm{Fe}_{2} \mathrm{O}_{3}$ 催化 $\mathrm{SMC}$ 反应

Scheme $10 \mathrm{Pd}-\gamma-\mathrm{Fe}_{2} \mathrm{O}_{3}$ catalyzed SMC reactions

Ghorbani-Choghamarani 课题组 ${ }^{[20]}$ 在 2016 年就另辟 蹊径，首先将纳米 $\mathrm{Fe}_{3} \mathrm{O}_{4}$ 用硅胶包裹，然后用 “linker” 化学键联钯 $(0)$ 配合物, 成功制备了一种新型负载型纳 米催化剂 Pd-ATBA-MNPs (Scheme 11). 与 $\mathrm{Pd}-\gamma-\mathrm{Fe}_{2} \mathrm{O}_{3}$ 相比，该催化剂不仅用量少 $(0.66 \sim 0.82 \mathrm{~mol} \%)$, 而且表 现出更加优异的底物普适性：氯代芳烃及四苯硼钠同样 有效，且 SMC 反应产率最高可达 $99 \%$. 其另一显著优 点是重复使用 6 次后催化活性仍高达 $95 \%$, 其重复利用 性能较传统的纳米钯催化剂有显著提升。

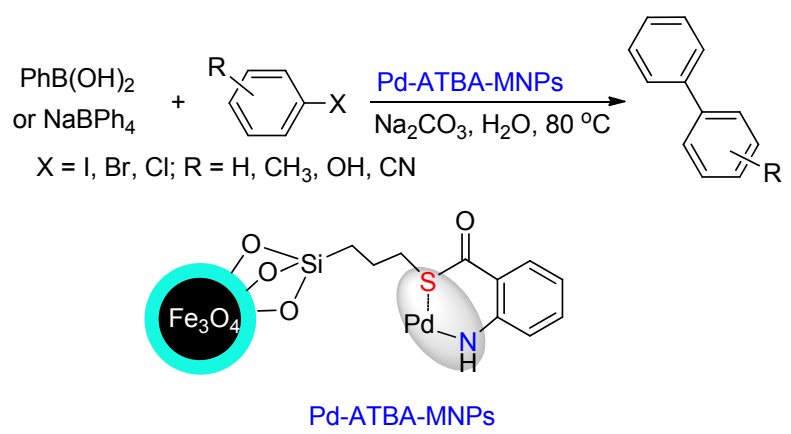

图式 11 Pd-ATBA-MNPs 催化 SMC 反应 Scheme 11 Pd-ATBA-MNPs catalyzed SMC reactions

2018 年, Naimi-Jamal 课题组 ${ }^{[21]}$ 则采用类似文献[20] 的方法 “化学键联” 钯(II)配合物, 制备了另一种新型的 纳米催化剂 $\mathrm{Fe}_{3} \mathrm{O}_{4} @ \mathrm{SiO}_{2} @$ GPTST@Pd (Scheme 12). 相 较于文献[20], 该催化剂用量较大 $(1.2 \mathrm{mmol} \%)$, 活性却 相对较低, 且只能活化苯硼酸. 尽管两个催化剂的配合 


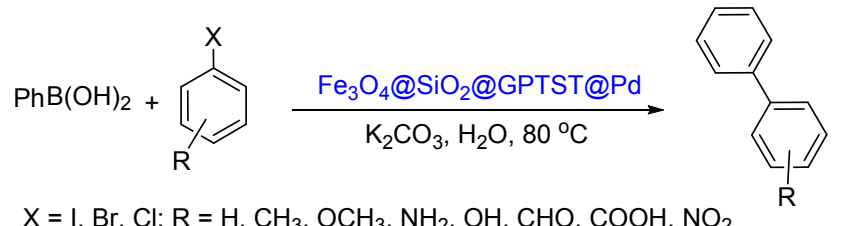

$\mathrm{X}=\mathrm{I}, \mathrm{Br}, \mathrm{Cl} ; \mathrm{R}=\mathrm{H}, \mathrm{CH}_{3}, \mathrm{OCH}_{3}, \mathrm{NH}_{2}, \mathrm{OH}, \mathrm{CHO}, \mathrm{COOH}, \mathrm{NO}_{2}$

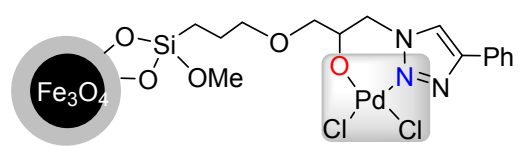

$\mathrm{Fe}_{3} \mathrm{O}_{4} @ \mathrm{SiO}_{2} @ G P T S T @ P d$

图式 $12 \mathrm{Fe}_{3} \mathrm{O}_{4} @ \mathrm{SiO}_{2} @ \mathrm{GPTST} @ \mathrm{Pd}$ 催化 SMC 反应 Scheme $12 \mathrm{Fe}_{3} \mathrm{O}_{4} @ \mathrm{SiO}_{2} @$ GPTST@Pd catalyzed SMC reactions

物结构不同, 但二者却表现出相近的重复使用率, 重复 使用 6 次后产率从 $98 \%$ 降至 95\%.

\section{$1.4 \mathrm{Pd}-\mathrm{Ru}$ 配合物催化剂}

$\mathrm{N}$-杂环卡宾(NHCs) 是近年来被广泛研究报道的一 类配体, 尤其是 Pd-NHCs 在 SMC 反应中表现出较高催 化活性 ${ }^{[22]} .2018$ 年, Choudhury 课题组 ${ }^{[23]}$ 开发了一种新 型的 “三明治” 结构配位聚合物 Ru-CP-Pd (Scheme 13). 尽管该双金属 $\mathrm{Ru} / \mathrm{Pd} / \mathrm{Ru}$ 催化剂以较高活性的 $\mathrm{Pd}-\mathrm{NHC}$ 为结构中心, 但在 $\mathrm{SMC}$ 反应中并没有明显的催化优势. 在 $90{ }^{\circ} \mathrm{C}$ 下, $0.1 \mathrm{~mol} \%$ 催化剂只能顺利活化碘及溴代芳 烃(产率 65\% 98\%), 而对氯代芳烃的活化能力则较差 (产率仅 $23 \%$ ). 此外, 该催化剂在连续使用 5 次后催化 活性下降较为明显, 产率仅为 $67 \%$.

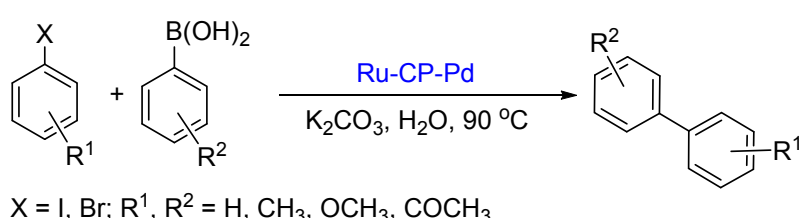

$X=I, B r ; R^{1}, R^{2}=H, C_{3}, \mathrm{OCH}_{3}, \mathrm{COCH}_{3}$
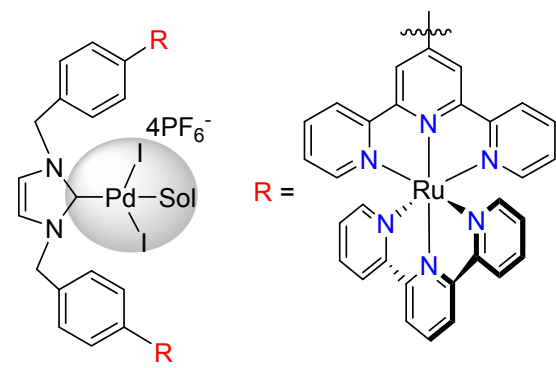

Ru-CP-Pd

图式 13 Ru-CP-Pd 催化 SMC 反应

Scheme 13 Ru-CP-Pd catalyzed SMC reactions

\section{$1.5 \mathrm{Pd}-\mathrm{Cu}-\mathrm{CeO}$ 纳米催化剂}

2018 年, Friedrich 等 ${ }^{[24]}$ 将 $\mathrm{Pd}_{0.04} \mathrm{Cu}_{0.04} \mathrm{Ce}_{0.92} \mathrm{O}_{2-\delta}$ 纳米 合金作为 SMC 反应的催化剂(Scheme 14). 这一催化剂 $(\mathrm{Pd} / \mathrm{Cu} / \mathrm{CeO}-\mathrm{TPAB})$ 的特殊点在于, $\mathrm{Pd} / \mathrm{Cu}$ 作为活性中心

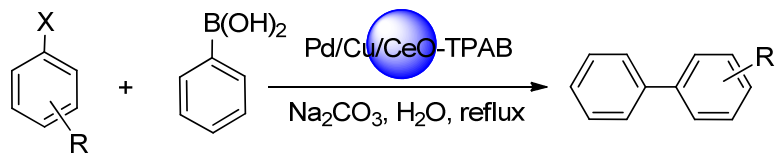

$\mathrm{X}=\mathrm{Br}, \mathrm{I} ; \mathrm{R}=\mathrm{H}, \mathrm{Me}, \mathrm{NH}_{2}, \mathrm{OCH}_{3}, \mathrm{CHO}, \mathrm{COCH}_{3}, \mathrm{NO}_{2}, \mathrm{CF}_{3}, \mathrm{~F}$

图式 $14 \mathrm{Pd} / \mathrm{Cu} / \mathrm{CeO}-\mathrm{TPAB}$ 催化 $\mathrm{SMC}$ 反应

Scheme $14 \mathrm{Pd} / \mathrm{Cu} / \mathrm{CeO}-\mathrm{TPAB}$ catalyzed SMC reactions

均匀分布在 $\mathrm{CeO}$ 晶格内, 形成统一的晶型结构, 从而增 加催化活性. 虽然该催化剂在用量较低的情况下 $(0.05$ $\mathrm{mol} \%$ ) 就能有效活化碘和溴代芳烃, 最高产率可达 $100 \%$, 但对空间位阻较大的溴代芳烃活化能力差(产率 48\%). 针对氯代芳烃而言, 即使增加了催化剂用量至 $0.23 \mathrm{~mol} \%$, 也只能活化连有两个 $\mathrm{NO}_{2}$ 的氯苯. 由于需 要四丙基溴化铵 (TPAB) 参与, 这一反应实际上是类非 均相反应，催化剂的活性在第二次使用时已降至 $60 \%$, 不具备重复使用性.

\section{2 均相催化 SMC 反应}

尽管均相反应中的催化剂难以分离和重复使用, 但 由于催化剂与反应底物处于同一相, 使反应底物易与活 性中心接触, 具有高活性和高选择性的优点. 正因为如 此, 科学家一直以来都在不断探索更加高效的均相催化 反应 ${ }^{[25]}$. 尤其是近年来, 以水为溶剂的均相催化反应更 是有机界的研究热点 ${ }^{[26]}$.

\section{1 含有聚乙二醇(PEG)的 Pd 催化剂}

PEG 不仅热稳定性好、难挥发、无毒、易于回收和 循环使用，且能溶解多种有机物和金属配合物，尤其具 有良好的水溶性. 目前, PEG 作为绿色介质已经被成功 应用到许多有机反应中 ${ }^{[27]}$ ，例如 Heck 反应、SMC 反应、 氧化还原反应、加成反应和不对称 aldo 反应等.

2018 年, 陈超等 ${ }^{[28]}$ 利用 PEG 的这一典型特点, 将 修饰的 DSPE-PEG 2000 纳米颗粒与 Pd-NHC 共聚, 制备了 一类具有两性特征的新型纳米催化剂 Pd-NHC/DSPE 2000 (Scheme 15). 这一催化剂在 $60{ }^{\circ} \mathrm{C}$ 和四丁基溴化铵 (TBAB) 参与下表现出优异的催化活性：仅 $0.01 \sim 0.1$ mol\% 用量就能高效活化溴和氯代芳烃(产率 $52 \%$ 99\%), 同时, 三(4-溴苯基)胺与芳基硣酸也能顺利进行 SMC 反应(产率 $80 \% \sim 93 \%$ ), 且官能团耐受性好. 值得 注意的是尽管是均相反应，但催化剂具有一定重复使用 能力, 水相重复使用 5 次后产率从 $95 \%$ 降至 $90 \%$, 催化 活性略有降低.

同年, Strassner 课题组 ${ }^{[29]}$ 则制备了一种连接 PEG 基 团的新型配合物 Pd-NHC/PEG (Scheme 16). 此催化剂 的结构特点是采用大空间位阻的 NHC 配体, 进一步提 升钯催化活性. 因此, 在 $100{ }^{\circ} \mathrm{C}$ 下, $0.1 \mathrm{~mol} \%$ 催化剂对 


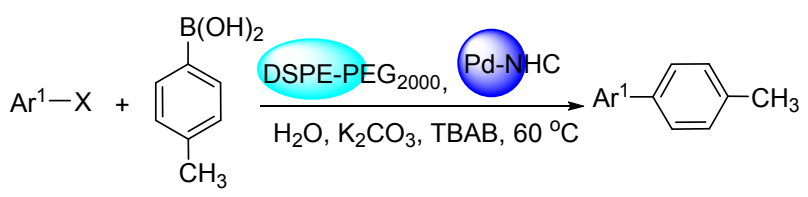

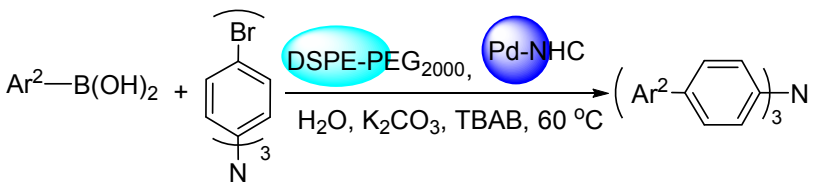
$\mathrm{X}=\mathrm{Br}, \mathrm{Cl} ; \mathrm{Ar}^{1}, \mathrm{Ar}^{2}=$ phenyl, pyridyl, pyrimidyl, thienyl

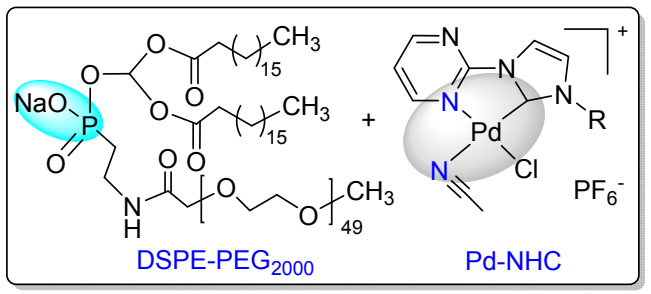

图式 $15 \mathrm{Pd}-\mathrm{NHC} / \mathrm{DSPE}_{2000}$ 催化 SMC 反应 Scheme 15 Pd-NHC/DSPE 2000 catalyzed SMC reactions

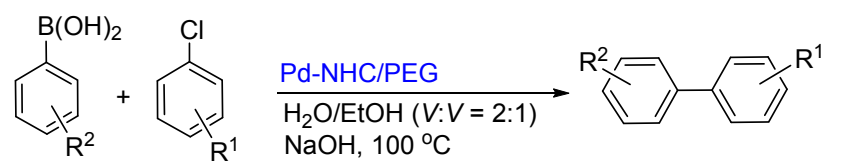
$\mathrm{R}^{1}=\mathrm{H}, \mathrm{CH}_{3}, \mathrm{OCH}_{3}, \mathrm{COCH}_{3}, \mathrm{NO}_{2} ; \mathrm{R}^{2}=\mathrm{H}, \mathrm{CH}_{3}$

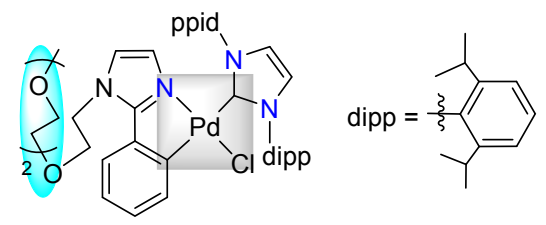

Pd-NHC/PEG

图式 $16 \mathrm{Pd}-\mathrm{NHC} / \mathrm{PEG}$ 催化 SMC 反应

Scheme 16 Pd-NHC/PEG catalyzed SMC reactions

多种不活泼的氯芳烃有活化优势，且不受空间位阻的影 响, $\mathrm{SMC}$ 反应产率可达 $48 \% \sim 100 \%$. 然而, 由于 $\mathrm{PEG}$ 基 团在配体分子中占比小, 因此催化剂的水溶性较差, 反 应是在 $\mathrm{H}_{2} \mathrm{O} / \mathrm{EtOH}(V: V=2: 1)$ 的混合溶剂中进行的.

\section{2 含有 $\beta-C D$ 的 $P d$ 催化剂}

$\beta$-环糊精 $(\beta-\mathrm{CD})$ 是由 7 个 $D-(+)$-吡喃型葡萄糖组成 的环状低聚物, 具有 “内腔疏水、外侧亲水” 的特点, 可 与多种无机及有机客体化合物形成包合物. 因而, $\beta-\mathrm{CD}$ 这一特殊的结构特性使其在催化领域受到了极大的关 注 $^{[30]}$.

2016 年, 杨睿课题组 ${ }^{[31]}$ 采用直接功能化 $\beta-\mathrm{CD}$ 的方 法, 即将氨基化的 $\beta-\mathrm{CD}$ 与 $\mathrm{Pd}(\mathrm{OAc})_{2}$ 络合, 成功制备了 一种新型催化剂 DAPH-Pd- $\beta$-CD (Scheme 17). 研究表 明该催化剂具有两大特点: 一方面, 在 $80{ }^{\circ} \mathrm{C}$ 反应温度

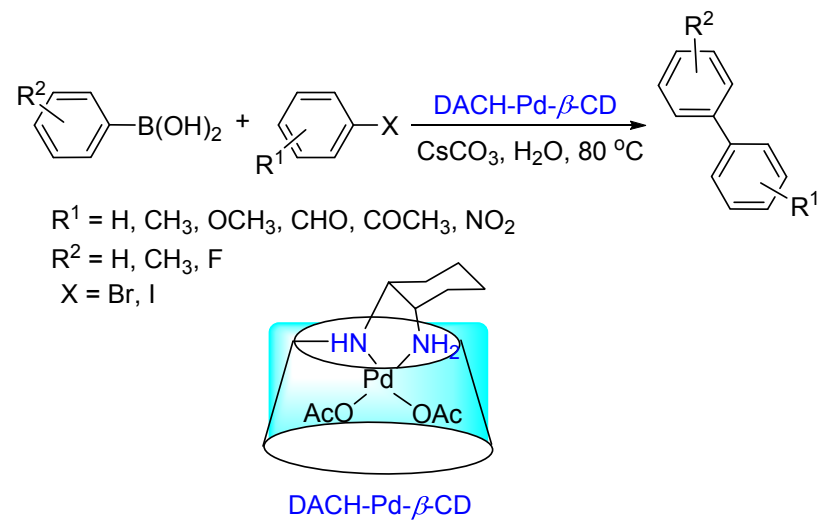

图式 17 DAPH-Pd- $\beta$-CD 催化 SMC 反应

Scheme 17 DAPH-Pd- $\beta$-CD catalyzed SMC reactions

下，尽管对氯代芳烃没有催化活性，但仅 $0.001 \mathrm{~mol} \%$ 的 用量就能顺利催化碘和溴代芳烃的 SMC 反应, 产率可 达 65\% 99\%, 很少有催化剂用量如此少却能达到如此 高的反应活性; 另一方面在重复使用能力上表现卓越, 水相中重复使用 10 次后其催化活性只降低了 $5 \%$.

同年, Pitchumani 课题组 ${ }^{[32]}$ 以离子化的吡啶修饰 $\beta-\mathrm{CD}$ 并络合 $\mathrm{Pd}(\mathrm{OAc})_{2}$, 制备了一种新型的催化剂 $\mathrm{Pd}(\mathrm{II}) @ \mathrm{Pyr} / \beta$-CD (Scheme 18). 由于采用离子型吡啶盐 作为配体, 进一步增大了该催化剂水溶性, 因而提高了 催化活性. 与 DAPH-Pd- $\beta-\mathrm{CD}$ 相比, 虽然用量相对较大 (0.5 mol\%), 但催化活性以及底物普适性更加优异, 不 仅能活化溴代芳烃，而且有效催化氯代芳烃(例如卤代 芳烃、芳杂卤代烃以及脂肪族卤代烃), 产率可达 72\% $95 \%$. 此外, 该催化剂表现出与 DAPH-Pd- $\beta-\mathrm{CD}$ 接近的 重复使用能力, 水相重复使用 6 次后催化剂活性没有明 显降低.

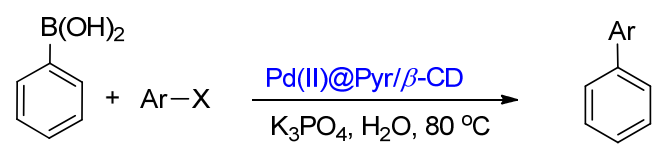

$\mathrm{X}=\mathrm{Br}, \mathrm{Cl} ; \mathrm{Ar}=$ phenethyl, adamantyl, phenyl, thienyl, pyridyl

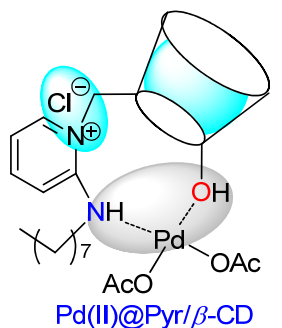

图式 $18 \mathrm{Pd}(\mathrm{II}) @ \mathrm{Pyr}: \beta-\mathrm{CD}$ 催化 SMC 反应

Scheme 18 Pd(II)@Pyr: $\beta$-CD catalyzed SMC reactions

2017 年, 吴勇课题组 ${ }^{[33]}$ 则独辟蹊径，用 “linker” 将 催化剂 $\beta-\mathrm{CD} / \mathrm{Pd}(\mathrm{OAc})_{2}$ 连接到氮化硼 $(\mathrm{BN})$ 载体上, 制备 了一种新型的负载型催化剂 h-BN@ $\beta-\mathrm{CD} @ \mathrm{Pd}(\mathrm{OAc})_{2}$ (Scheme 19). 在 $70{ }^{\circ} \mathrm{C}$ 下, 该催化剂能高效催化溴代芳 
烃的 SMC 反应(产率可达 79\% 99\%)，且官能团耐受性 好, 但对氯代芳烃没有催化活性. 其另一个明显的结构 特征是 BN 载体在反应温度下溶于水, 但室温下却不溶 于水. 因此, 催化剂易于回收, 而且具有高度的稳定性 和重复使用率，连续使用 9 次后产率从 $99 \%$ 仅降至 97\%, 基本保持原有的催化活性. 此外, 作者使用该催化剂通 过 SMC 反应成功制备了抗消炎药物, 并进行了克量级 的 SMC 反应等方面的尝试, 同样收到很好的效果. 因 此, 该项研究为这一类型的催化剂在推广应用以及规模 化生产上提供了可能.
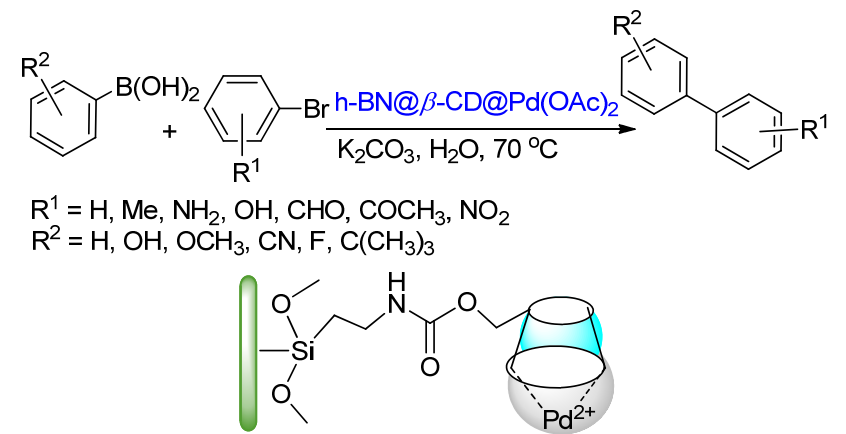

h-BN@ $\beta-\mathrm{CD} @ \mathrm{Pd}(\mathrm{OAc})_{2}$

图式 $19 \mathrm{~h}-\mathrm{BN} @ \beta-\mathrm{CD} @ \mathrm{Pd}(\mathrm{OAC})_{2}$ 催化 SMC 反应 Scheme 19 h-BN@ $\beta$-CD $@ \mathrm{Pd}(\mathrm{OAC})_{2}$ catalyzed SMC reactions

以上研究中, $\mathrm{Pd}(\mathrm{OAc})_{2}$ 与 $\beta-\mathrm{CD}$ 结合所形成的催化 剂在水中催化 SMC 反应, 实际上兼具了均相催化反应 “催化剂活性和选择性高” 以及非均相催化反应 “催化 剂用量少、能重复使用” 的优点, 尤其是文献[33], 更是 具有 “均相催化, 多相回收” 的优势 ${ }^{[22 b]}$, 大大缩小了均 相与非均相反应之间的界限.

\section{3 含有离子型配体的 $\mathrm{Pd}$ 催化剂}

目前, 在水相中实现均相 SMC 催化反应最为直接 的方法就是采用水溶性配体与钯络合, 形成水溶性催化 剂. 因此, 这一类型催化剂的研发也特别引人注目. 相 较于阴离子和阳离子配体而言, 两性离子型配体因自身 是含有两种相反电荷的基团, 水溶性更优, 同时也增加 了在水相中进行均相催化反应的可能性.

2016 年, Das 课题组 ${ }^{[34]}$ 用 $\mathrm{SO}_{3} \mathrm{Na}$ 基团修饰 2-羟基苯 席夫碱配体, 同时与钯络合形成溶于水的新型四配位催 化剂 Pd-Salen 配合物(Scheme 20). 在室温下, $0.1 \mathrm{~mol} \%$ 催化剂顺利催化碘或溴代芳烃的 SMC 反应, 产率达 $62 \% \sim 98 \%$; 可是, 当反应温度升高到 $100{ }^{\circ} \mathrm{C}$, 催化剂 用量增大到 $1 \mathrm{~mol} \%$, 氯代芳烃的 SMC 产率依然较低, 使用十六烷基三甲基澳化铵(CTAB)为相转移催化剂后, 产率才得到较大程度的改善. 值得注意的是, 该催化剂 表现出较高的底物普适性以及官能团耐受性, 例如吡 啶、噻吩以及嘧啶等结构的芳杂卤代烃或芳杂硼酸都能
顺利地进行 SMC 反应.

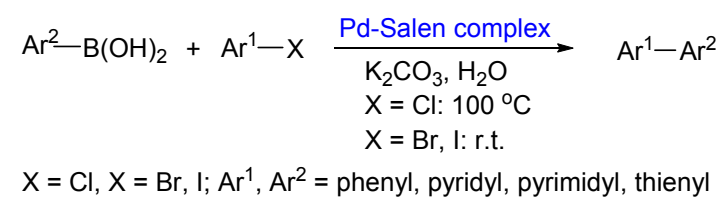

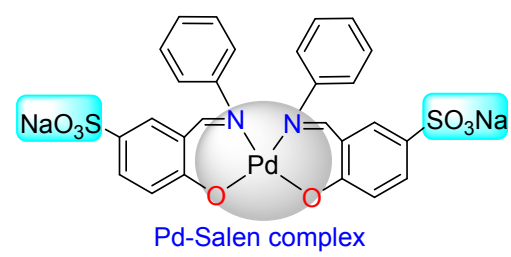

图式 20 Pd-Salen 配合物催化 SMC 反应

Scheme 20 Pd-Salen complex catalyzed SMC reactions

同年, 孙亚光课题组 ${ }^{[35]}$ 则报道了一种新型的钯钴 双金属配合物 $\mathrm{CoPd}_{2}(\mathrm{HBPDC})_{2} \mathrm{Cl}_{4}\left(\mathrm{H}_{2} \mathrm{O}\right)_{4}$ (Scheme 21). 该催化剂具有突出的结构特点,一方面, 双金属 $\mathrm{Pd} / \mathrm{Co} / \mathrm{Pd}$ 协同催化的同时, 以 2,2-联吡定为配体有利于 电子传递, 因此进一步提高 SMC 反应的催化活性; 另 一方面，配体上连有 $\mathrm{COOH}$ 基团，在碱性 $\mathrm{K}_{2} \mathrm{CO}_{3}$ 条件下 为 $\mathrm{COOK}$ 盐, 因而催化剂在反应条件下具有良好的水 溶性. 这一离子型配体催化剂与 DAPH-Pd- $\beta$-CD 的催化 能力接近, 仅 $0.001 \mathrm{~mol} \%$ 的用量就能顺利催化碘或溴代 芳烃的 SMC 反应，而且 SMC 产率可达 $67 \% \sim 98 \%$, 同 样对氯代芳烃没有催化活性; 但遗憾的是, 该催化剂不 具备重复使用能力.

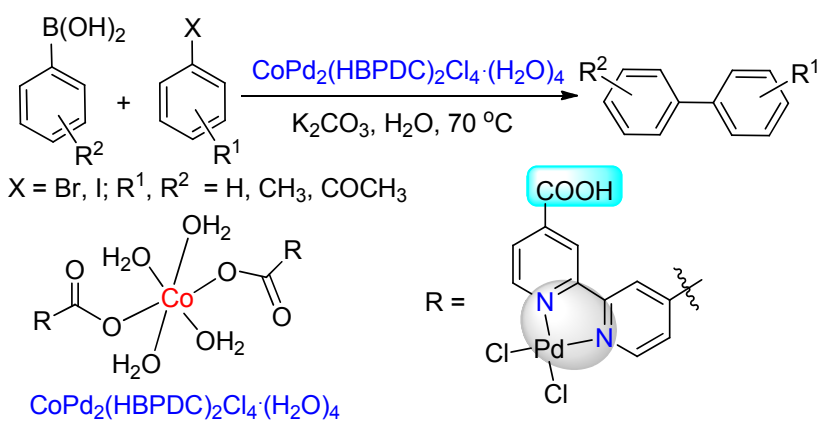

图式 $21 \mathrm{CoPd}_{2}(\mathrm{HBPDC})_{2} \mathrm{Cl}_{4}\left(\mathrm{H}_{2} \mathrm{O}\right)_{4}$ 催化 $\mathrm{SMC}$ 反应 Scheme $21 \mathrm{CoPd}_{2}(\mathrm{HBPDC})_{2} \mathrm{Cl}_{4}\left(\mathrm{H}_{2} \mathrm{O}\right)_{4}$ catalyzed SMC reactions

2018 年, $\mathrm{Kinfe}$ 课题组 ${ }^{[36]}$ 通过 $\mathrm{PdCl}_{2}$ 与具有螯形结构 的 SNS 配体络合, 制备了一系列新型钯(II)阳离子配合 物钯螯形配合物(Scheme 22). 在 TBAB 参与下, 0.5 $\mathrm{mol} \% \mathrm{~A}, \mathbf{B}, \mathbf{C}$ 和 D 都能顺利催化 4 -溴苯甲醚和苯嗍酸的 SMC 反应，产率可达 91\% 94\%. 其中，D 催化剂体现 出更为高效的催化活性，无论溴代芳烃还是氯代芳烃都 能高效活化，产率可达 77\% 91\%，且官能团耐受性广. 由于反应是在 $120{ }^{\circ} \mathrm{C}$ 下完成的, 需密封加压, 因此反应 条件较为苛刻. 虽然该催化剂表现出一定的重复使用能 
力, 但水相在使用 3 次后催化活性已有较为明显的降低.

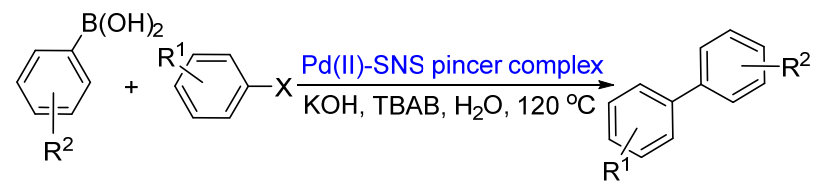

$\mathrm{R}^{1}=\mathrm{CH}_{3}, \mathrm{OCH}_{3}, \mathrm{COCH}_{3}, \mathrm{NH}_{2}, \mathrm{CN}, \mathrm{NO}_{2}$

$\mathrm{R}^{2}=\mathrm{H}, \mathrm{OCH}_{3}, \mathrm{Cl}, \mathrm{CHO}, \mathrm{NO}_{2}, \mathrm{CH}\left(\mathrm{CH}_{3}\right)_{2}$

$\mathrm{X}=\mathrm{Br}, \mathrm{Cl}$

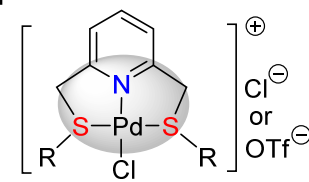

$\mathrm{A}: \mathrm{R}=\mathrm{Ph} ; \mathrm{B}: \mathrm{R}=p-\mathrm{BrC}_{6} \mathrm{H}_{4}$

C: $\mathrm{R}=4-{ }^{t} \mathrm{BuC}_{6} \mathrm{H}_{4} ; \mathrm{D}: \mathrm{R}=s-\mathrm{Bu}$

$\mathrm{Pd}(\mathrm{II})-\mathrm{SNS}$ pincer complex

图式 22 钯螯形配合物催化 SMC 反应

Scheme 22 Pd(II)-SNS pincer complex catalyzed SMC reactions

膦配体是 SMC 反应早期就开始研究应用的钯催化 剂配体, 具有与钯配位好、催化活性高的优点. 相较于 NHCs 配体, 膦配体的催化性能更优. 然而, 由于膦配 体的毒性大于氮配体, 使得其在绿色化学领域的应用受 到了一定的限制. 尽管如此, 人们还是特别关注水溶性 两性离子膦配体的研发.

Reddy 课题组 ${ }^{[37]}$ 开拓了两种新型的两性离子钯配 合物 $\mathrm{Pd}\left(\mathrm{HL}_{1}\right)\left(\mathrm{PPh}_{3}\right) \mathrm{Cl}_{2}$ 和 $\mathrm{Pd}\left(\mathrm{HL}_{2}\right)\left(\mathrm{PPh}_{3}\right) \mathrm{Cl}_{2}$ (Scheme 23). 其中, 作者较系统地研究了 $\mathrm{Pd}\left(\mathrm{HL}_{1}\right)\left(\mathrm{PPh}_{3}\right) \mathrm{Cl}_{2}$ 催化剂, 其结构特点是, 一方面, 两性离子配体结构增加了其水 溶性, 有利于提高催化活性; 另一方面, 使用膦配体进 一步提高催化能力. 与文献[36]相比, 该催化剂不需要 相转移催化剂的参与就能大幅度降低 SMC 的反应温度 $\left(70{ }^{\circ} \mathrm{C}\right)$, 而且还大大扩展了底物的适用范围, 尤其是杂 环溴代芳烃和苄基溴代烃都能与芳基及杂环芳基硼酸 或氟嗍盐进行 $\mathrm{C}\left(\mathrm{sp}^{2}\right)-\mathrm{C}\left(\mathrm{sp}^{2}\right)$ 和 $\mathrm{C}\left(\mathrm{sp}^{3}\right)-\mathrm{C}\left(\mathrm{sp}^{2}\right)$ 偶联, $\mathrm{SMC}$ 产率可达 65\% 98\%. 但遗憾的是, 该催化剂对氯 代芳烃没有催化活性.

$$
\begin{aligned}
& \mathrm{Ar}^{1}-\mathrm{Br}+\mathrm{Ar}^{2}-\mathrm{Y} \underset{\mathrm{K}_{2} \mathrm{CO}_{3}, \mathrm{H}_{2} \mathrm{O}, 70^{\circ} \mathrm{C}}{\mathrm{Pd}(\mathrm{HL})\left(\mathrm{PPh}_{3}\right) \mathrm{Cl}_{2}} \mathrm{Ar}{ }^{1}-\mathrm{Ar}^{2} \\
& \mathrm{Y}=\mathrm{B}(\mathrm{OH})_{2}, \mathrm{BF}_{3} \mathrm{~K} \\
& \mathrm{Ar}^{1}=\text { phenyl, benzyl, quinolyl, pyridyl, pyrimidyl, thienyl } \\
& \mathrm{Ar}^{2}=\text { thienyl, benzothiophenyl, indolyl, dibenzofuran }
\end{aligned}
$$

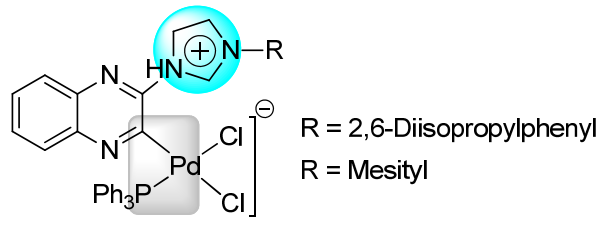

$\mathrm{Pd}(\mathrm{HL})\left(\mathrm{PPh}_{3}\right) \mathrm{Cl}_{2}$

图式 $23 \mathrm{Pd}(\mathrm{HL})\left(\mathrm{PPh}_{3}\right) \mathrm{Cl}_{2}$ 催化 $\mathrm{SMC}$ 反应

Scheme $23 \mathrm{Pd}(\mathrm{HL})\left(\mathrm{PPh}_{3}\right) \mathrm{Cl}_{2}$ catalyzed SMC reactions

2017 年, Canovese 等 ${ }^{[38]}$ 报道了一种新型的两性离子
催化剂 Pd-NHC/TPPTS (Scheme 24). 其结构的显著特 点就是集膦配体(TPPTS) 和氮配体(NHC)于同一催化剂 中. 与 $\mathrm{Pd}(\mathrm{HL})\left(\mathrm{PPh}_{3}\right) \mathrm{Cl}_{2}$ 相比, 尽管该催化剂也是两性离 子结构, 同时 $\mathrm{SO}_{3} \mathrm{Na}$ 基团进一步增加了催化剂的水溶 性, 但还需在 TBAB 的参与下完成反应. 在最佳反应温 度 $80{ }^{\circ} \mathrm{C}$ 下, $0.1 \mathrm{~mol} \%$ 催化剂对溴代芳烃催化活性高, $\mathrm{SMC}$ 产率可达 95\% 98\%, 但官能团的耐受能力有限. 同样，该催化剂对氯代芳烃没有催化活性.
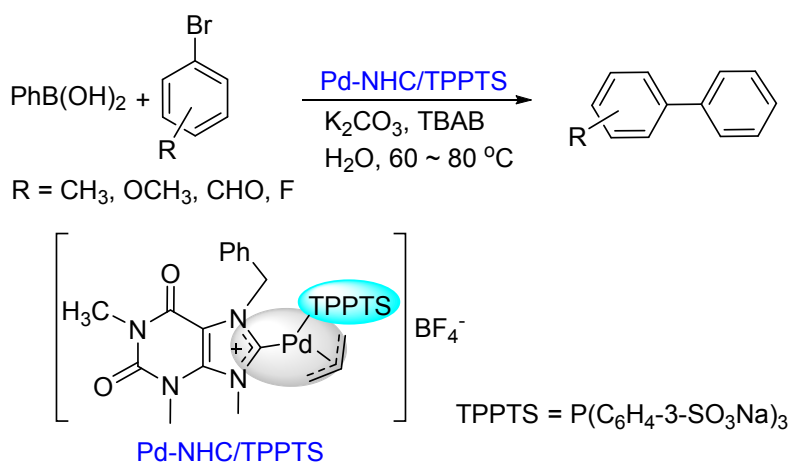

图式 24 Pd-NHC/TPPTS 催化 SMC 反应

Scheme 24 Pd-NHC/TPPTS catalyzed SMC reactions

同年, Lee 课题组 ${ }^{[39]}$ 则以连有咪唑鎓盐的碳供体与 三环已基膦 $\left(\mathrm{PCy}_{3}\right)$ 为配体制备了一种新型的两性离子配 合物 $\mathrm{Pd}-\mathrm{PCy}_{3} /$ imidazolium (Scheme 25). 该催化剂在室 温下水溶性差，却在反应温度 $80{ }^{\circ} \mathrm{C}$ 时具有很好的水溶 性. 相较于 Pd-NHC/TPPTS, 尽管该催化剂用量较大(2 $\mathrm{mol} \%$ ), 但在顺利活化氯代芳烃上优势明显, SMC 产率 可达 $62 \% \sim 100 \%$, 只是对空间位阻较大的底物催化活 性较弱. 值得注意的是, 反应需在 $1 \mathrm{~g}$ 用量的 TBAB 参 与下才能进行. 一方面 TBAB 是相转移催化剂, 另一方 面又是催化剂在水中的稳定剂，不仅大幅度改善催化活 性，同时又提升了催化剂的重复使用率. 因此，水相在 连续使用 9 次后依然能保持原有催化活性.
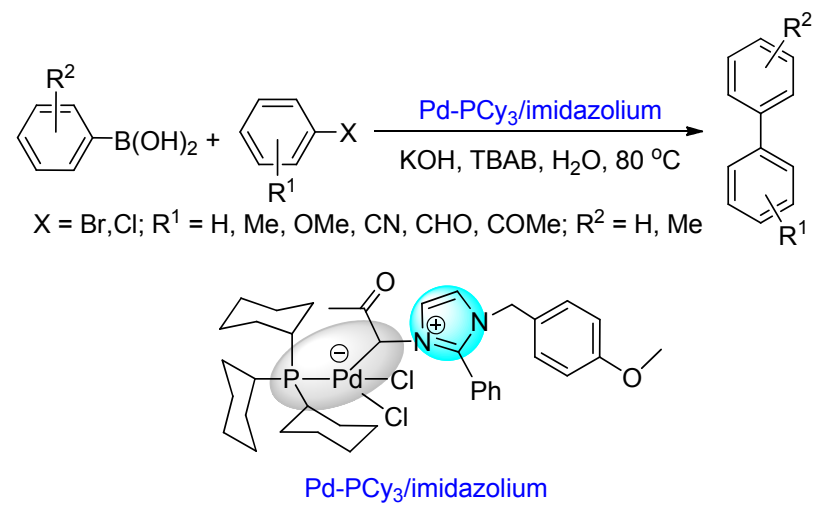

图式 $25 \mathrm{Pd}^{-\mathrm{PCy}_{3}} /$ imidazolium 催化 $\mathrm{SMC}$ 反应 Scheme $25 \mathrm{Pd}-\mathrm{PCy}_{3} /$ imidazolium catalyzed SMC reactions 2016 年，段新红等 ${ }^{[40]}$ 报道了一种新型两性离子型 
膦配体(Scheme 26). 在室温下, 这一配体与 $\mathrm{PdCl}_{2}$ 原位 络合形成稳定的六元环结构, 并表现出优异的催化活 性, $0.15 \mathrm{~mol} \%$ 用量就能高效催化碘或澳代芳烃的 SMC 反应，底物普适性广，官能团耐受性强，对空间位阻不 敏感，底物间芳基-芳基、芳基-杂芳基、杂芳基-杂芳基 偶联都能顺利进行，产率可达 76\% 93\%. 尽管反应条 件温和, 但对氯代芳烃没有催化活性. 此外, 还利用此 催化剂通过 “一锅法” 对 2,5-二溴吡定连续进行 Ph$\mathrm{B}(\mathrm{OH})_{2}$ 和 $4-\mathrm{CH}_{3} \mathrm{OC}_{6} \mathrm{H}_{4} \mathrm{~B}(\mathrm{OH})_{2}$ 的 $\mathrm{SMC}$ 反应, 总产率达 $61 \%$, 表明该催化剂具有很好的区域选择性.
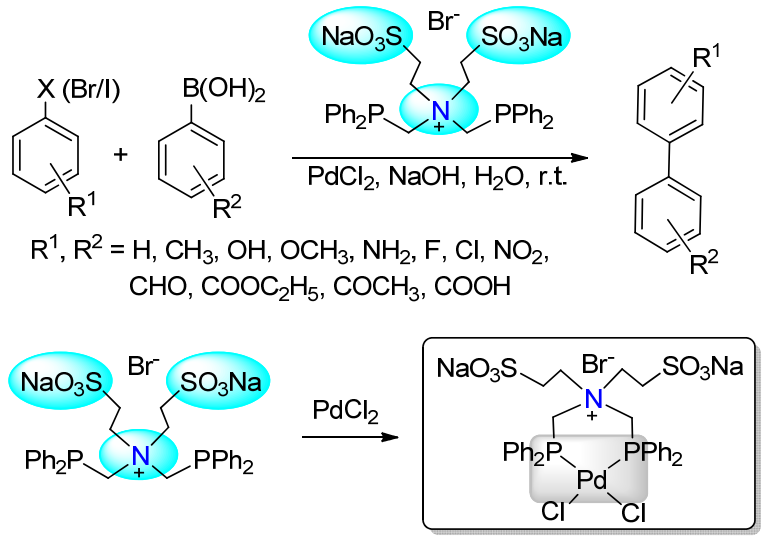

图式 26 新型两性膦配体 Pd 配合物催化 SMC 反应 Scheme 26 Pd complex with a zwitterionic phosphine ligand catalyzed SMC reactions

随后，段新红课题组 ${ }^{[41]}$ 又报道了采用 “一步法” 实 现两个不同溴代芳烃的硼化/SMC 反应的研究(Scheme 27). 这一反应的突出优势是采用一个催化剂同时实现 嗍化和 SMC 两个钯催化反应, 是步骤简约的反应; 使 用 $2 \mathrm{~mol} \%$ 催化剂、等物质的量溴代芳烃及廉价 $\mathrm{B}_{2}(\mathrm{OH})_{4}$, 又是原子经济的反应. 研究表明, 在 $100{ }^{\circ} \mathrm{C}$ 反应温度 下, 水溶性 $\mathrm{Na}_{2} \mathrm{PdCl}_{4} / \mathrm{PCy}_{3} \mathrm{HBF}_{4}$ 首先活化的是相对富电 子的澳代芳烃, 并与 $\mathrm{B}_{2}(\mathrm{OH})_{4}$ 原位生成芳基硼酸, 与此 同时催化另一个相对缺电子的卤代芳烃并进行 SMC 反 应. 值得注意的是, 两个溴代烃在反应过程中不产生自

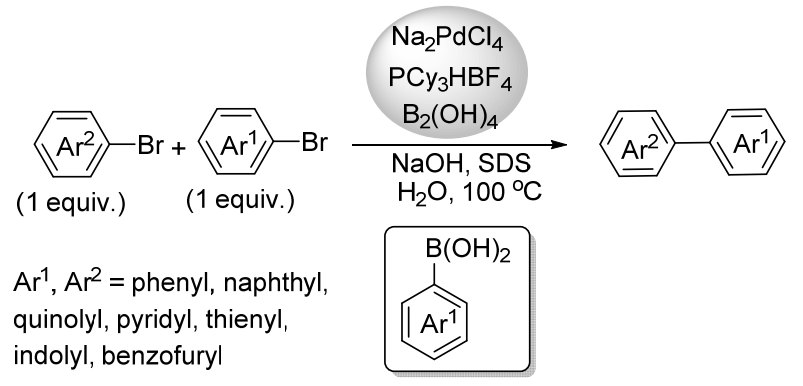

图式 27 一步法实现两个不同溴代芳烃的嗍化/SMC 反应 Scheme 27 One-step borylation/SMC of two distinct arylbromides
身偶联副产物，而且底物适用性广，官能团耐受性强， 底物间芳基-芳基、芳基-杂芳基、杂芳基-杂芳基偶联 都能顺利进行. 此外, 该方法在 2,5-二溴吡啶的单芳基 化以及 3,5-二澳吡啶的连续芳基化时都表现出优秀的区 域选择性，表明其在制备复杂联苯类化合物上具有优 势.

\section{3 非离子型表面活性剂参与的均相反应}

非离子表面活性剂在水中和有机溶剂中都有较好 的溶解性，且不易电离，稳定性好，尤其是在室温下能 与一定比例的金属催化剂在水中形成纳米胶束, 大大促 进了催化剂进行均相反应的速度与选择性, 因此, 近年 来受到科学家的关注和青睐.

1996 年, Saito 等 ${ }^{[42]}$ 首次报道了 $(\mathrm{dppf}) \mathrm{NiCl}_{2} / \mathrm{BuLi}$ 体 系在 1,4-二氧六环溶剂中高效催化氯代芳烃与苯嗍酸的 SMC 反应, 但自此后该体系在水介质中催化 SMC 的研 究一直没有突破性进展. 2015 年, Lipshutz 课题组 ${ }^{[43]}$ 发 现，非离子表面活性剂 TPGS-750-M 与(dippf) $\mathrm{NiCl}_{2}$ 或 (dppf) $\mathrm{NiCl}_{2}$ 在水中能形成纳米胶束催化剂(Scheme 28). 与文献[42]对比发现, 这两种新型镍纳米胶束催化剂用 量(1 3 mol\%)是文献[42]中的近十分之一，但催化活性 却大大提高了, 不仅能在接近室温下 $\left(45{ }^{\circ} \mathrm{C}\right)$ 进行 $\mathrm{SMC}$ 反应，同时大大扩展了底物的适用范围，例如卤代芳烃 $(\mathrm{Cl} 、 \mathrm{Br}$ 和 $\mathrm{I})$ 及磺酸芳基酯都能顺利进行 $\mathrm{SMC}$ 反应; 喼 化试剂除 $\mathrm{ArB}(\mathrm{OH})_{2}$ 外, ArBpin 和 ArBMIDA 同样有效, 底物间芳基-芳基、芳基-杂芳基、杂芳基-杂芳基偶联 都能顺利进行(产率 $72 \% \sim 96 \%$ ), 且官能团耐受性好. 另一点值得关注的是: 由于这两种镍纳米胶束催化剂在 水中具有很好稳定性，因此，水相回收并重复使用 6 次 后依然能保持原有的催化活性.

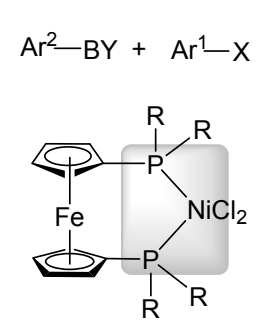

$$
\begin{gathered}
\frac{\text { (dippf) } \mathrm{NiCl}_{2} \text { or (dppf) } \mathrm{NiCl}_{2}}{\operatorname{MeMgBr}(3 \mathrm{~mol} \%), \mathrm{K}_{3} \mathrm{PO}_{4}, 45^{\circ} \mathrm{C}} \mathrm{Ar}^{1}-\mathrm{Ar}^{2} \\
\underset{\mathrm{TPGS}-750-\mathrm{M}(w=2 \%)}{ }
\end{gathered}
$$

$\mathrm{BY}=\mathrm{B}(\mathrm{OH})_{2}, \mathrm{BMIDA}, \mathrm{Bpin} ; \mathrm{X}=\mathrm{Cl}, \mathrm{Br}, \mathrm{I}$, OTf $A r^{1}, A^{2}=$ phenyl, naphthyl, quinolyl, pyridyl, thiazolyl, thienyl, benzothiophenyl, indolyl, benzofuran, benzoxazolyl

(dippf) $\mathrm{NiCl}_{2}: \mathrm{R}={ }^{i} \mathrm{Pr}$ (dppf) $\mathrm{NiCl}_{2}: \mathrm{R}=\mathrm{Ph}$<smiles>Cc1c(C)c(OC(=O)CCC(=O)OCCOC(C)(C)C)c(C)c2c1CCC(C)([As])O2</smiles>

图式 $28 \mathrm{NiCl}_{2} / \mathrm{TPGS}-750-\mathrm{M}$ 催化 SMC 反应 Scheme $28 \mathrm{NiCl}_{2} / \mathrm{TPGS}-750-\mathrm{M}$ catalyzed SMC reactions

2014 年, Giri 等 ${ }^{[44]}$ 使用 $\mathrm{CuI} / 2-\mathrm{P}(t-\mathrm{Bu})_{2} \mathrm{PhNMe}_{2}$ 催化 剂在 DMF/1,4-二氧六环混合溶剂中进行 SMC 反应. 由于 
存在催化活性弱(只能催化碘代芳烃)及难回收等缺陷, 因此, 人们对铜催化体系进行 SMC 的研究一直较少 ${ }^{[45]}$. 2016 年, Lipshutz 课题 组 $^{[46]}$ 将 $0.02 \% \mathrm{Pd}(\mathrm{OAc})_{2}$ 与 $\mathrm{Cu}(\mathrm{OTf})_{2} / 2-\mathrm{P}(t-\mathrm{Bu})_{2} \mathrm{PhNMe}_{2}$ 组合, 制备了一种新型的纳 米合金 $(\mathrm{Cu} / \mathrm{ppmPd} \mathrm{NPs})$, 并探索其与 TPGS-750-M 在水 中形成的纳米胶束对 SMC 反应的影响(Scheme 29). 与 文献[44]对比发现, 这一催化体系具有明显优势, 如反 应温度显著降低、反应时间明显缩短, 但遗憾的是催化 能力依然止步于碘代芳烃, 而且底物的适用性与官能团 的耐受性并没有太大的改善. 此外, 这一纳米胶束催化 剂表现出类似于文献[43]的重复使用能力, 水相回收并 重复使用 6 次后产率从 $92 \%$ 降到 $91 \%$, 基本保持原有的 催化活性.

$$
\mathrm{Ar}^{2}-\mathrm{B}(\mathrm{OH})_{2}+\mathrm{Ar}^{1}-\frac{\mathrm{Cu} / \mathrm{ppmPd} \mathrm{NPs}(10 \mathrm{~mol} \%)}{\mathrm{Na}_{2} \mathrm{CO}_{3}, \mathrm{H}_{2} \mathrm{O}, 45^{\circ} \mathrm{C}} \longrightarrow \mathrm{Ar}^{1}-\mathrm{Ar}^{2}
$$

图式 $29 \mathrm{Cu} / \mathrm{ppmPd}$ NPs/TPGS-750-M 催化 SMC 反应 Scheme $29 \mathrm{Cu} / \mathrm{ppmPd}$ NPs/TPGS-750-M catalyzed SMC reactions

2018 年, Lipshutz 课题组 ${ }^{[47]}$ 继续探索 TPGS-750-M 与另一种钯催化剂 $\operatorname{Pd}(\text { EvanPhos })_{2}$ 在水中形成纳米胶束 并催化 SMC 反应(Scheme 30). 将 Pd(EvanPhos) $)_{2}$ 在有机 溶剂中进行 SMC 反应与这一纳米胶束对比发现, 胶束 催化剂用量更少, 反应速度更快, 产率更高, 而且碘、溴 及氯代芳烃都能顺利进行 SMC 反应. 与文献[43]类似, 该反应底物普适性广，官能团耐受性强，尤其是仅 0.05 $\mathrm{mol} \%$ 用量催化剂就能成功制备安塞曲匹(Anacetrapib) 药物的中间体, 产率为 $91 \%$. 遗憾的是, 这一纳米胶束 重复使用能力差, 重复使用 2 次后催化活性已从 $93 \%$ 降 至 $90 \%$.

2017 年, Patel 课题组 ${ }^{[48]}$ 将表面活性剂 Triton X-100 与 $\mathrm{Pd}(\mathrm{OAc})_{2} / r a c-B I-D I M E$ 在水中形成一种新型纳米胶 束催化剂, 并对此进行 SMC 反应的研究(Scheme 31). 与文献[47]相比, 在 $70{ }^{\circ} \mathrm{C}$ 下该催化剂只能活化溴代芳 烃, 但用量较少、底物普适性更广; 并能顺利实现底物 间芳基-芳基、芳基-杂芳基、杂芳基-杂芳基偶联，产率 可达 $84 \% \sim 98 \%$, 官能团耐受性强. 研究还发现, 以质 量分数为 $5.0 \times 10^{-4}$ 的催化剂与质量分数为 $1 \%$ Triton X-100 在水中成功制备了 50 克量级的手性药物分子 11-beta-HSD-1 Inhibitor, 产率为 97\%. 这一规模化的合 成无疑为推广该类催化体系进行工业生产提供了前景.

$$
\mathrm{Ar}^{2}-\mathrm{B}(\mathrm{OH})_{2}+\mathrm{Ar}^{1}-\mathrm{X} \quad \frac{\text { EvanPhos }}{\stackrel{\mathrm{K}_{2} \mathrm{PO}_{4}, \mathrm{H}_{2} \mathrm{O}, 45^{\circ} \mathrm{C}}{\mathrm{TPGS}-750-\mathrm{M}(w=2 \%)}} \mathrm{Ar}^{1}-\mathrm{Ar}^{2}
$$

$\mathrm{Ar}^{1}, \mathrm{Ar}^{2}=$ phenyl, pyridyl, pyrimidyl, indolyl, benzofuryl $\mathrm{X}=\mathrm{Cl}, \mathrm{Br}, \mathrm{I}$

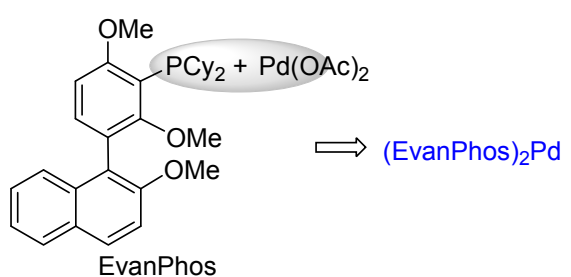

图式 $30 \mathrm{Pd}(\text { EvanPhos })_{2} / \mathrm{TPGS}-750-\mathrm{M}$ 催化 SMC 反应 Scheme $30 \mathrm{Pd}(\text { EvanPhos })_{2} /$ TPGS-750-M catalyzed SMC reactions

$$
\begin{aligned}
& \mathrm{Ar}^{2}-\mathrm{Y}+\mathrm{Ar}-\mathrm{Br} \frac{\text { rac-BI-DIME }}{\frac{\mathrm{H}_{2} \mathrm{O}, \mathrm{K}_{2} \mathrm{CO}_{3}, 70^{\circ} \mathrm{C}}{\text { Triton } \mathrm{X}-100(w=0.5 \%)}} \mathrm{Ar}^{1}-\mathrm{Ar}^{2} \\
& \mathrm{Ar}^{1}=\text { phenyl, naphthyl, indolyl, pyrimidyl, pyridyl, } \\
& \quad \text { quinolyl, naphthyl, furyl } \\
& \mathrm{Ar}^{2}=\text { naphthyl, quinolyl, benzofuryl, phenyl } \\
& \mathrm{Y}=\mathrm{B}(\mathrm{OH})_{2}, \text { Bpin }
\end{aligned}
$$

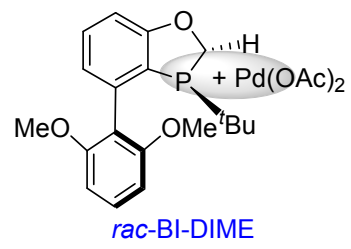

图式 $31 \mathrm{Pd}(\mathrm{OAc})_{2} / \mathrm{rac}-\mathrm{BI}-\mathrm{DIME} /$ Triton X-100 催化 SMC 反应 Scheme $31 \quad \mathrm{Pd}(\mathrm{OAc})_{2} / \mathrm{rac}$-BI-DIME/Triton $\mathrm{X}-100$ catalyzed SMC reactions

遗憾的是，作者并没有对该催化剂重复使用能力进行研 究.

Beverina 课题组 ${ }^{[49]}$ 报道了另一种广泛使用的表面 活性剂 Kolliphor-EL 与 $\mathrm{PdCl}_{2}(\mathrm{dtbpf})$ 在水中形成纳米胶 束催化剂(Scheme 32). 对比研究中发现, Kolliphor-EL 的氧气敏感度远低于 TPGS-750-M 及 Triton X-100, 因 此, 在空气环境下由 Kolliphor-EL 形成的钯纳米胶束粒 子表现出更高的 $\mathrm{SMC}$ 产率. 与 $\mathrm{PdCl}_{2}(\mathrm{dtbpf})$ 在 $\mathrm{DMF}$ 中 的 $\mathrm{SMC}$ 反应 ${ }^{[50]}$ 相比, 该纳米胶束在水中的反应条件更 温和, 反应速度更快, 并对溴代芳烃及二溴代芳烃都具 有高效的催化活性. 作者正是利用该催化体系的氧敏感 度低及在室温下高效催化 SMC 反应的独特优势, 制备 了多种富有挑战性的半导体材料, 并收到很好的效果. 然而，该体系在重复使用 2 次后产率从 $90 \%$ 降至 70\%, 不具备重复使用能力.

\section{4 其它类型的均相反应}

2017 年, Schmitzer 课题组 ${ }^{[51]}$ 探索了 $\mathrm{Pd}(\mathrm{OAc})_{2}$ /二甲 双胍盐酸盐(metformin $\cdot \mathrm{HCl}$ )催化的 $\mathrm{SMC}$ 反应(Scheme 


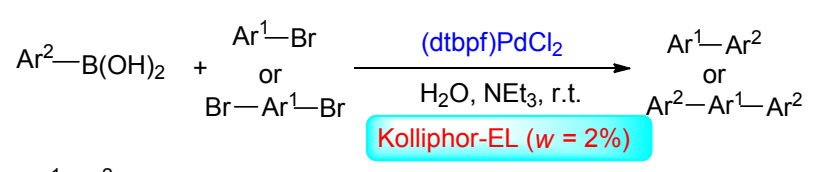

$A r^{1}, A r^{2}=$ phenyl, naphthyl, quinolyl, thienyl

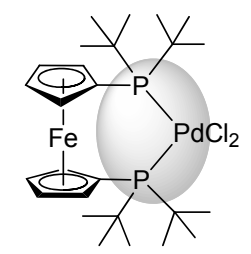

(dtbpf) $\mathrm{PdCl}_{2}$

图式 32 (dtbpf)PdCl $\mathrm{Pd}_{2} / \mathrm{Kolliphor-EL}$ 催化 $\mathrm{SMC}$ 反应 Scheme 32 (dtbpf) $\mathrm{PdCl}_{2} /$ Kolliphor-EL catalyzed SMC reactions

33). 研究表明, 在 $100{ }^{\circ} \mathrm{C}$ 下, $\mathrm{Pd}(\mathrm{OAc})_{2}$ 与二甲双胍原位 生成一种新型钯配合物, $0.25 \mathrm{~mol} \%$ 用量就能顺利催化 溴代芳烃，但对氯代芳烃没有催化作用.

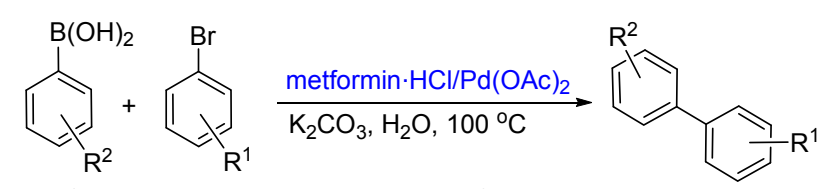

$\mathrm{R}^{1}=\mathrm{CH}_{3}, \mathrm{CHO}, \mathrm{COCH}_{3}, \mathrm{CN}, \mathrm{NH}_{2} ; \mathrm{R}^{2}=\mathrm{CH}_{3}, \mathrm{COCH}_{3}$

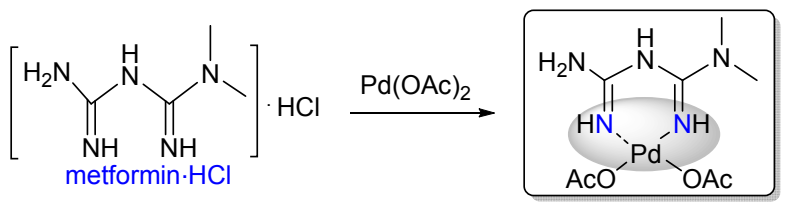

图式 $33 \mathrm{Pd}(\mathrm{OAC})_{2} / \mathrm{metformin} \bullet \mathrm{HCl}$ 催化 $\mathrm{SMC}$ 反应 Scheme $33 \mathrm{Pd}(\mathrm{OAC})_{2} /$ metformin $\bullet \mathrm{HCl}$ catalyzed SMC reactions

Lee 课题组 ${ }^{[52]}$ 则独辟蹊径, 将富含赖氨酸的多肽 $\left(\mathrm{YC}_{7}\right)$ 制备成为 $\alpha$-螺旋次生肽结构, 此结构在 $\mathrm{Pd}^{2+}$ 参与 下能均匀自聚成含有桥连结构的球状纳米二维肽 YC7@ $\mathrm{Pd}^{2+}$ (Scheme 34). 研究发现, 在 $80{ }^{\circ} \mathrm{C}$ 下, 该催 化剂自动解聚成溶于水的纳米钯一肽配合物, 并顺利地 催化碘代芳烃与苯嗍酸的 SMC 反应, 但对溴代和氯代 芳烃没有催化作用; 当体系温度回到室温时, 催化剂则 又重新自聚为原来的结构, 给 “多相回收催化剂” 带来 了便利, 但对于回收的催化剂能否再利用没有进一步的 研究和报道.

\section{3 总结与展望}

自从铃木一宫浦偶联反应问世以来，人们就对该反 应进行了坚持不解的研究和探索, 尤其是近二十年以 来, 以绿色溶剂水为介质的铃木一宫浦偶联反应的研究 已取得了突破性进展, 主要针对活泼卤代烃如碘代烃、
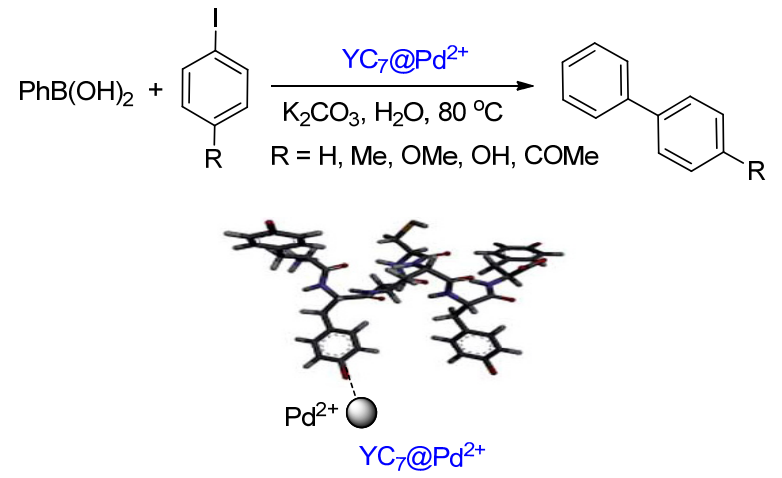

图式34 YC7@ $\mathrm{Pd}^{2+}$ 催化 SMC 反应

Scheme 34 YC7@ $\mathrm{Pd}^{2+}$ catalyzed SMC reactions

溴代烃及不活泼氯代烃等亲电试剂与芳基硼酸、芳基频 哪醇酯及芳基硼酸盐等亲核试剂的偶联反应进行了大 量的研究, 探索出不少能在水溶剂中实现铃木一宫浦偶 联反应的高效、高选择性非均相或均相催化体系.

尽管以水为溶剂的铃木一宫浦偶联反应目前已取得 了丰硕成果, 但同时也面临着许多挑战. 如何实现绿色 化及工业化氯代芳烃在水相中的铃木一宫浦偶联反应是 目前的难题之一. 开发廉价的 $\mathrm{Ni}, \mathrm{Cu}$ 和 $\mathrm{Fe}$ 等来代替 $\mathrm{Pd}$ 催化剂更是该反应面临的一大挑战.

总之, 迎接挑战, 发掘更加绿色廉价的可回收均相 催化剂和能低成本大规模生产的载体来逐步消除均相 与非均相催化剂之间的界限是铃木一宫浦偶联反应的发 展方向, 对绿色有机化学的发展和工业化生产都具有深 远意义.

\section{References}

[1] (a) Hirner, J. J.; Shi, Y.; Blum, S. A. Accounts Chem. Res. 2011, 44, 603.

(b) Rosen, B. M.; Quasdorf, K. W.; Wilson, D. A.; Zhang, N.; Resmerita, A.-M.; Garg, N. K.; Percec, V. Chem. Rev. 2011, 111, 1346.

(c) Jana, R.; Pathak, T. P.; Sigman, M. S. Chem. Rev. 2011, 111, 1417.

(d) Molnár, Á. Chem. Rev. 2011, 111, 2251.

(e) Han, F.-S. Chem. Soc. Rev. 2013, 42, 5270.

(f) Valente, C.; Calimsiz, S.; Hoi, K. H.; Mallik, D.; Sayah, M.; Organ, M. G. Angew. Chem. Int. Ed. 2012, 51, 3314.

(g) Knappke, C. E. I.; Grupe, S.; Gartner, D.; Corpet, M.; Gosmini, C.; Wangelin, A. J. Chem.-Eur. J. 2014, 20, 6828.

(g) Knappke, C. E. I.; Grupe, S.; Gartner, D.; Corpet, M.; Gosmini, C.; Wangelin, A. J. Chem.-Eur. J. 2014, 20, 6828.

(h) Pan, C.; Liu, M.; Duan, X. Chin. J. Org. Chem. 2015, 35, 472 (in Chinese).

(潘春娇, 刘敏, 段新红, 有机化学, 2015, 35, 472.)

[2] Miyaura, N.; Yanagi, T.; Suzuki, A. Synth Commun. 1981, 513.

[3] (a) Shaughnessy, K. H.; Booth, R. S. Org. Lett. 2001, 3, 2757.

(b) Anderson, K. W.; Buchwald, S. L. Angew. Chem., Int. Ed. 2005, 44, 6173 .

(c) Suzuki, A. Angew. Chem., Int. Ed. 2011, 50, 6723.

(d) Ge, S.; Hartwig, J. F. Angew. Chem., Int. Ed. 2012, 51, 12837.

(e) Mondal, M.; Bora, U. Green Chem. 2012, 14, 1873.

(f) Ramgren, S. D.; Hie, L.; Ye, Y.; Garg, N. K. Org. Lett. 2013, 15, 3950 . 
(g) Molander, G. A.; Argintaru, O. A. Org. Lett. 2014, 16, 1904.

[4] (a) Ennis, D. S.; McManus, J.; Wood-Kaczmar, W.; Richardson, J.; Smith, G. E.; Carstairs, A. Org. Process Res. Dev. 1999, 3, 248.

(b) Zhou, S.-L.; Xu, L.-W.; Xia, C.-G.; Li, J.-W.; Li, F.-W. Chin. J. Org. Chem. 2004, 24, 1501 (in Chinese).

(周少林, 徐利文, 夏春谷, 李经纬, 李福伟, 有机化学, 2004, 24, 1501.)

(c) Jiang, H.; Zhang, M.; Zhang, L.; Chen Y.; Zhu, N.; Song, L.; Deng, H. Chin. J. Org. Chem. 2017, 37, 2399 (in Chinese). (蒋海芳, 张敏, 张丽, 陈雅丽, 朱宁, 宋力平, 邓红梅, 有机化 学, 2017, 37, 2399.)

[5] (a) Engberts, J. B. F. N.; Blandamer, M. J. Chem. Commun. 2001, $18,1701$.

(b) Sinou, D. Adv. Synth. Catal. 2002, 344, 221.

(c) Klijn, J. E.; Engberts, J. B. F. N. Nature 2005, 435, 746.

(d) Li, C. J. Chem. Rev. 2005, 105, 3095.

(e) Shaughnessy, K. H.; DeVasher, R. B. Curr. Org. Chem. 2005, 9, 585 .

(f) Li, C. J., Chen, L. Chem. Soc. Rev. 2006, 35, 68

(g) Zhang, J.; Yin, H.; Han, S. Chin. J. Org. Chem. 2012, 32, 1429 (in Chinese).

(张敬先，殷慧清，韩世清，有机化学, 2012, 32, 1429.)

[6] Polshettiwar, V.; Decottignies, A.; Len, C.; Fihri, A. ChemSusChem 2010, 3, 502 .

[7] (a) Maluenda, I.; Navarro, O. Molecules 2015, 20, 7528. (b) Chatterjee, A.; Ward, T. R. Catal. Lett. 2016, 146, 820.

[8] Li, X.; Xu, H.; Zhou, J.; Yan, G.; Zhang, L.; Zhuo, S. Chin. J. Org Chem. 2018, 38, 1917 (in Chinese).

(李晓微，许海芬，周晋，间格，张雷，禚淑萍，有机化学，2018, 38 , 1917.)

[9] (a) Joucla, L.; Batail, N.; Djakovitch, L. Adv. Synth. Catal. 2010, $352,2929$.

(b) Moncea, O.; Poinsot, D.; Fokin, A. A.; Schreiner, P. R.; Hierso, J.-C. Chem CatChem 2018, 10, 2915.

(c) Ge, C.; Sang, X.; Yao, W.; Zhang, L.; Wang, D. Green Chem. 2018, 20, 1805 .

(d) Xu, Z.; Yu, X.; Sang, X.; Wang, D. Green Chem. 2018, 20, 2571.

(e) Ye, D.; Huang, R.; Zhu, H.; Zou, L.-H.; Wang, D. Org. Chem. Front. 2019, 6, 62.

(f) Ye, D.; Pan, L.; Zhu, H.; Jin, L.; Miao, H.; Wang, D. Mater. Chem. Front. 2019, 3, 216

(g) Qiu, Y.; Zhang, Y.; Jin, L.; Pan, L.; Du, G.; Ye, D.; Wang, D. Org. Chem. Front. 2019, 6, 3420.

(h) Hu, W.; Zhang, Y.; Zhu, H.; Ye, D.; Wang, D. Green Chem. 2019, 21, 5345 .

(i) Yang, Q.; Zhang, Y.; Zeng, W.; Duan, Z.-C.; Sang, X.; Wang, D. Green Chem. 2019, 21, 5683.

[10] Gogoi, N.; Bora, U.; Borah, G.; Gogoi P. K. Appl. Organomet. Chem. 2016, e3686.

[11] Shabbir, S.; Lee, S.; Lim, M.; Lee, H.; Ko, H.; Lee, Y.; Rhee, H. J. Organomet. Chem. 2017, 846, 296.

[12] Chen, J.; Zhang, J.; Zhu, D.; Li, T. Appl. Organomet. Chem. 2018, 32, e3996.

[13] Rathod, P. V.; Jadhav, V. H. Tetrahedron Lett. 2017, 58, 1006.

[14] Rostamnia, S.; Doustkhah, E.; Zeynizadeh, B. Microporous Mesoporous Mater. 2016, 222, 87.

[15] Destito, P.; Sousa-Castillo, A.; Couceiro, J. R.; López, F.; Correa-Duarte, M. A.; Mascareñas, J. L. Chem. Sci. 2019, 10, 2598

[16] Kim, Y.-O.; You, J. M.; Jang, H.-S.; Choi, S. K.; Jung, B. Y.; Kang, O.; Kim, J. W.; Lee, Y.-S. Tetrahedron Lett. 2017, 58, 2149.

[17] Holzer, C.; Dupé, A.; Peschel, L. M.; Belaj, F.; Mösch-Zanetti, N. C. Eur. J. Inorg. Chem. 2018, 568.

[18] Mandegani, Z.; Asadi, M.; Asadi, Z. Appl. Organomet. Chem. 2016, $30,657$.

[19] Paul, D.; Rudra, S.; Rahman, P.; Khatua, S.; Pradhan, M.; Chatterjee, P. N. J. Organomet. Chem. 2018, 871, 96.

[20] Ghorbani-Choghamarani, A.; Tahmasbi, B.; Moradi P. Appl. Or- ganomet. Chem. 2016, 30, 422.

[21] Dadras, A.; Naimi-Jamal, M. R.; Moghaddam, F. M.; Ayati, S. E. Appl. Organomet. Chem. 2018, 32, e3993.

[22] (a) Ni, C.; Shen, A.; Cao, Y.; Ye, X. Chin. J. Org. Chem. 2014, 34, 278 (in Chinese).

(倪晨, 沈安, 曹育才, 叶晓峰, 有机化学, 2014, 34, 278.)

(b) Wang, W.; Cui, L.; Sun, P.; Shi, L.; Yue, C.; Li, F. Chem. Rev. 2018, $118,9843$.

[23] Mondal, M.; Joji, J.; Choudhury, J. J. Chem. Sci. 2018, 130, 83

[24] Mpungose, P. P.; Sehloko, N. I.; Maguire, G. E.; Friedrich, H. B. New J. Chem. 2017, 41, 13560.

[25] (a) Tang, W.; Zhang, X. Chem. Rev. 2003, 103, 3029.

(b) Wang, D.; Yu, X.; Ge, B.; Miao, H.; Ding, Y. Chin. J. Org Chem. 2015, 35, 676 (in Chinese).

(王大伟, 余晓丽, 葛冰洋, 苗红艳, 丁玉强, 有机化学, 2015, 35, 676.)

(c) Guo, N.; Zhu, S. Chin. J. Org. Chem. 2015, 35, 1383 (in Chinese).

(郭娜, 朱守非, 有机化学, 2015, 35, 1383.)

(d) Chen, S.; Yang, W.; Yao, Y.; Yang, X.; Deng, Y.; Yang, D. Chin. J. Org. Chem. 2018, 38, 2534 (in Chinese).

(陈姝琪，杨文，姚永祺，杨新，邓颖颖，杨定乔，有机化学， 2018, 38, 2534.)

(e) Hu, X.; Yang, B.; Yao, W.; Wang, D. Chin. J. Org. Chem. 2018, 38, 3296 (in Chinese).

(胡昕宇，杨伯斌，姚玮，王大伟，有机化学，2018，38，3296.)

(f) Liu, N.; Chao, F.; Liu, M.-G.; Huang, N.-Y.; Zou, K.; Wang, L. J. Org. Chem. 2019, 84, 2366.

(g) Liu, M.-G.; Liu, N.; Xu, W.-H.; Wang, L. Tetrahedron 2019, 75, 2748.

[26] (a) Jamwal, N.; Gupta, M.; Paul, S. Green Chem. 2008, 10, 999.

(b) McLaughlin, M. P.; McCormick, T. M.; Eisenberg, R.; Holland, P. L. Chem. Commun. 2011, 47, 7989.

(c) Lipshutz, B. H.; Isley, N. A.; Moser, R.; Ghorai, S.; Leuser, H.; Taft, B. R. Adv. Synth. Catal. 2012, 354, 3175.

(d) Debnath, K.; Pathak, S.; Pramanik, A. Tetrahedron Lett. 2013 $54,4110$.

(e) Yuan, Z.-F.; Zhao, W.-N.; Liu, Z.-P.; Xu, B.-Q. J. Catal. 2017, 353,37 .

[27] (a) Chandrasekhar, S.; Narsihmulu, C.; Shameem Sultana, S.; Ramakrishna Reddy, N. Org. Lett. 2002, 4, 4399.

(b) Feu, K. S.; de la Torre, A. F.; Silva, S.; F de Moraes Junior, M. A.; Corrêa, A. G.; Paixão, M. W. Green Chem. 2014, 16, 3169.

(c) Tiwari, A. R.; Bhanage, B. M. Green Chem. 2016, 18, 144.

(d) Xiao, L.; Dai, F.; Li, Z.; Jing, X.; Kong, J.; Liu, G. Chin. J. Org. Chem. 2019, 39, 648 (in Chinese).

(肖立伟, 戴富才, 李政, 景学敏, 孔洁, 刘光仙, 有机化学, 2019, 39, 648.)

[28] Chen, C.; Zheng, Q.; Ni, S.; Wang, H. New J. Chem. 2018, 42, 4624.

[29] Schroeter, F.; Soellner, J.; Strassner, T. Organometallics 2018, 37 , 4267.

[30] (a) Shen, H.; Ji, H. Chin. J. Org. Chem. 2011, 31, 791 (in Chinese). (沈海民, 纪红兵, 有机化学, 2011, 31, 791.)

(b) Kaboudin, B.; Abedi, Y.; Yokomatsu, T. Org. Biomol. Chem. 2012, 10, 4543 .

(c) Yang, Z.; Zhang, X.; Yao, X.; Fang, Y.; Chen, H.; Ji, H. Tetrahedron 2016, 72, 1773 .

[31] Guo, Y.; Li, J.; Shi, X.; Liu, Y.; Xie, K.; Liu, Y.; Jiang, Y.; Yang, B.; Yang, R. Appl. Organomet. Chem. 2017, 31, e3592.

[32] Khan, R. I.; Pitchumani, K. Green Chem. 2016, 18, 5518.

[33] Ma, X.; Lv, G.; Cheng, X.; Li, W.; Sang, R.; Zhang, Y.; Wang, Q.; Hai, L.; Wu, Y. Appl. Organomet. Chem. 2017, 31, e3854.

[34] Shahnaz, N.; Puzari, A.; Paul, B.; Das, P. Catal. Commun. 2016, 86 , 55.

[35] You, L.-X.; Liu, H.-J.; Cui, L.-X.; Ding, F.; Xiong, G.; Wang, S.-J.; Ren, B.-Y.; Dragutan, L.; Dragutan, V.; Sun, Y.-G. Dalton Trans. 2016, 45,18455 
[36] Fiebor, A.; Tia, R.; Makhubela, B. C. E.; Kinfe, H. H. Beilstein J. Org. Chem. 2018, 14, 1859.

[37] Ramakrishna, V.; Reddy N. D. Dalton Trans. 2017, 46, 8598.

[38] Scattolin, T.; Canovese, L.; Visentin, F.; Paganelli, S.; Canton, P.; Demitri, N. Appl. Organomet. Chem. 2017, e4034.

[39] Lee, J.-Y.; Tzeng, R.-J.; Wang, M.-C.; Lee, H. M. Inorg. Chim. Acta 2017, 464, 74

[40] Qiu, P.; Zhao, J. Y.; Shi, X.; Duan, X. H. New J. Chem. 2016, 40, 6568.

[41] Xu, S. D.; Sun, F. Z.; Deng, W. H.; Hao, H.; Duan, X. H. New J. Chem. 2018, 42, 16464.

[42] Saito, S.; Sakai, M.; Miyaura, N. Tetrahedron Lett. 1996, 37, 2993.

[43] Handa, S.; Slack, E. D.; Lipshutz, B. H. Angew. Chem., Int. Ed. 2015, 54, 11994.

[44] Gurung, S. K.; Thapa, S.; Kafle, A.; Dickie, D. A.; Giri, R. Org. Lett. 2014, 16, 1264.

[45] (a) Wang, L.; Xie, Y.-B.; Huang, N.-Y.; Yan, J.-Y.; Hu, W.-M.; Liu, M.-G.; Ding, M.-W. ACS Catal. 2016, 6, 4010. (b) Wang, L.; Xie, Y.-B.; Huang, N.-Y.; Zhang, N.-N.; Li, D.-J.; Hu, Y.-L.; Liu, M.-G.; Lia, D.-S. Adv. Synth. Catal. 2017, 359, 779.

[46] Handa, S.; Smith, J. D.; Hageman, M. S.; Gonzalez, M.; Lipshutz, B. H. ACS Catal. 2016, 6, 8179.

[47] Landstrom, E. B.; Handa, S.; Aue, D. H.; Gallou, F.; Lipshutz, B. H. Green Chem. 2018, 20, 3436

[48] Patel, N. D.; Rivalti, D.; Buono, F. G.; Chatterjee, A.; Qu, B.; Braith, S.; Desrosiers, J.-N.; Rodriguez, S.; Sieber, J. D.; Haddad, N.; Fandrick, K. R.; Lee, H.; Yee, N. K.; Busacca, C. A.; Senanayake, C. H. Asian J. Org. Chem. 2017, 6, 1285.

[49] Mattiello, S.; Rooney, M.; Sanzone, A.; Brazzo, P.; Sassi, M.; Beverina, L. Org. Lett. 2017, 19, 654.

[50] Sajith, A. M.; Abdul Khader, K. K.; Muralidharan, A.; Ali Padusha, M. S.; Nagaswarupa, H. P. J. Heterocycl. Chem. 2015, 52, 1748.

[51] Fortun, S.; Beauclair P.; Schmitzer, A. R. RSC Adv. 2017, 7, 21036.

[52] Kim, S.; Cho, H.-J.; Lee, N.; Lee, Y.-S.; Shin, D.-S.; Lee, S.-M. RSC Adv. 2017, 7, 33162. 